This is the author's final, peer-reviewed manuscript as accepted for publication. The publisher-formatted version may be available through the publisher's web site or your institution's library.

\title{
The network level reproduction number for infectious diseases with both vertical and horizontal transmission
}

Ling Xue, Caterina Scoglio

\section{How to cite this manuscript}

If you make reference to this version of the manuscript, use the following information:

Xue, L., \& Scoglio, C. (2013). The network level reproduction number for infectious diseases with both vertical and horizontal transmission. Retrieved from http://krex.ksu.edu

\section{Published Version Information}

Citation: Xue, L., \& Scoglio, C. (2013). The network level reproduction number for infectious diseases with both vertical and horizontal transmission. Mathematical Biosciences, 243(1), 67-80.

Copyright: ( 2013 Elsevier Inc.

Digital Object Identifier (DOI): doi:10.1016/j.mbs.2013.02.004

Publisher's Link: http://www.sciencedirect.com/science/article/pii/S0025556413000503

This item was retrieved from the K-State Research Exchange (K-REx), the institutional repository of Kansas State University. K-REx is available at http://krex.ksu.edu 


\title{
The network level reproduction number for infectious diseases with both vertical and horizontal transmission
}

\author{
Ling Xue*, Caterina Scoglio \\ Department of Electrical \& Computer Engineering, \\ Kansas State University, U.S. 66506
}

\begin{abstract}
A wide range of infectious diseases are both vertically and horizontally transmitted. Such diseases are spatially transmitted via multiple species in heterogeneous environments, typically described by complex meta-population models. The reproduction number, $R_{0}$, is a critical metric predicting whether the disease can invade the meta-population system. This paper presents the reproduction number for a generic disease vertically and horizontally transmitted among multiple species in heterogeneous networks, where nodes are locations, and links reflect outgoing or incoming movement flows. The metapopulation model for vertically and horizontally transmitted diseases is gradually formulated from two species, two-node network models. We derived an explicit expression of $R_{0}$, which is the spectral radius of a matrix reduced in size with respect to the original next generation matrix. The reproduction number is shown to be a function of vertical and horizontal transmission parameters, and the lower bound is the reproduction number for horizontal transmission. As an application, the reproduction number and its bounds for the Rift Valley fever zoonosis, where livestock, mosquitoes, and humans are the involved species are derived. By computing the reproduction number for different scenarios through numerical simulations, we found the reproduction number is affected by livestock movement rates only when parameters are heterogeneous across nodes. To summarize, our study contributes the reproduction number for vertically and horizontally transmitted diseases in heterogeneous networks. This explicit expression is easily adaptable to specific infectious diseases, affording insights into disease evolution.
\end{abstract}

Keywords:

reproduction number, vertical and horizontal transmission, heterogeneous networks, Rift Valley fever, multiple species

\section{Introduction}

Communicable diseases are readily transmitted from one region to another $[1,2]$. Population travel continues to influence the temporal and spatial spread of infectious diseases $[1,3]$. Observation of the introduction of infectious agents resulting in spatial spreading of effective infections in different locations at different times [3], revealed great economic losses, many animal and human cases, and deaths. Noteworthy examples include the fourteenth century plague in Europe $[1,4]$ and the sixteenth century smallpox epidemic in the New World [1]. More recent epidemics, including HIV/AIDS and West Nile virus in North America [5], and SARS in Asia [6], show infections spreading over vast regions and even jumping continents [7].

\footnotetext{
* Corresponding Author

Email addresses: lxue@ksu.edu (Ling Xue), caterina@k-state.edu (Caterina Scoglio)
} 
Many communicable diseases are propagated by two distinct mechanisms: vertical and horizontal transmission [8]. Vertical transmission occurs when infection is passed from mother to a portion of offspring [8, 9], often transmitted by insect eggs and/or plant seeds [10]. A variety of diseases are transmitted vertically and horizontally, including the human diseases rubella, hepatitis B, Chagas disease, and AIDS [10, 11]. Vertical transmission is a proven factor in the size and persistence of Rift Valley fever (RVF) epidemic [12]. The prevalence of vertical transmission establishes it as a crucial biological mechanism [11], potentially affecting infectious spreading in elaborate ways [13]. Therefore, vertical transmission acts to maintain the spread of infection $[13,14]$. The logical complement of vertical transmission is horizontal transmission. For animal and human diseases [10], horizontal transmission is often through direct or indirect contact with infectious hosts or infectious vectors, such as biting insects [10].

Spatially structured models, such as meta-population models or multiple-patch models are widely used in epidemiological modeling to capture the effect of space [15]. Meta-population models describe systems containing spatially discrete sub-populations connected by the movement of individuals between a set of patches or nodes $[16,17]$. Modeling the dynamics of large metapopulations is complex, presenting challenges during analysis [18]. One approach considers the mobility of individuals between discrete regions [18], creating a directed network where nodes represent locations and links are movements between locale [18]. The importance of tracking mobility rates and movement patterns is highlighted in the foot-and-mouth outbreak of 2001 in the United Kingdom [7]. There, infected cattle were widely distributed before the movement ban was announced [19], prompting the necessary development of a transportation network capturing the spatial spread of foot-and-mouth disease [7].

Numerical tools are widely used to obtain quantitative results and analytic tools are used to understand model behaviors [3]. The reproduction number, $R_{0}$, defined as the average number of new infected individuals produced by one infectious individual, in a population with only susceptibles [20], is arguably the most important quantity in communicable disease modeling [20]. Theoretically, $R_{0}$ plays an important role in analyzing the dynamics of an epidemic [20]. It is a quantity commonly used to estimate the dynamics of emerging infectious diseases at the beginning of an outbreak, aiding in the design of control strategies for established infections [20]. The next generation method developed by [21], [22, Chapter 5] and popularized by [23] is one of many methods applied to compute the reproduction number for compartmental models. This method manages matrix size by including only infected and asymptomatically infected states [24]. The next generation matrix relates the number of new cases in various generations and provides the basis of defining and computing the reproduction number $[20]$.

The very little work on the reproduction number for meta-populations with vertical transmission we encountered included the modeling of horizontal and vertical transmission dynamics of a parasite with two differential equations [25]. In this special case, the reproduction number is the sum of the reproduction numbers for both types of transmission, and does not hold for a more complicated situation, such as in the model [26], where the next generation matrices for the two types of transmission are not both scalars. As far as we know, an insightful explicit expression of $R_{0}$ for multiple species meta-population model with complex transmission has not yet been presented.

This paper presents the computation of the reproduction number and its bounds for compartmental models considering diseases with complex transmission. We consider meta-populations consisting of discrete, well-mixed subpopulations. We assume that individuals move between different nodes and the disease can be transmitted within a node. An $n$-node compartmental model incorporates $h$ species, of which $g$ species transmit a disease both vertically and horizontally and other species only transmit horizontally. All sojourn times are taken to be exponentially distributed, and vertical transmission is restricted to the egg stage with exponential duration. Presented here is a general network-level model applicable when studying the temporal-spatial propagation of an infectious disease with multi-species, vertical and horizontal transmission, where the reproduction number is derived as a function of the 
two types of transmission parameters. Finally, the exact value and bounds of the reproduction number for the RVF meta-population model are computed and factors affecting the reproduction number are analyzed. We found the upper bound depends on both horizontal and vertical transmission, while the lower bound is determined solely by horizontal transmission.

The contribution of our work is summarized as follows:

1. An explicit expression of the reproduction number considering vertical and horizontal transmission in a general multi-species, meta-population model is derived.

2. This formula for the reproduction number is applied to an RVF meta-population model to compute $R_{0}$ and its bounds.

3. Numerical simulations show that livestock movement rates only affect $R_{0}$ for heterogeneous networks relative to disease parameters.

Our work facilitates computation of the exact reproduction number in a meta-population model with complex disease transmission.

The paper is organized as follows. Section 2 describes the next generation matrix approach used to derive an explicit expression of the reproduction number, and presents the general meta-population model beginning with two species, two-node network models, as well as computing the reproduction number. In Section 3, we apply our $R_{0}$ formula to the RVF meta-population model, computing $R_{0}$ and its bounds. The effects of livestock movement, heterogeneities of parameters, and the size of a network on the reproduction number are also studied through simulations. Section 4 provides a summary and discussion of mathematical derivations and simulation results.

\section{The reproduction number for diseases with both vertical and horizontal transmission}

One frequently used method computes the reproduction number as the spectral radius of the next generation matrix [22, Chapter 5], [27, 20]. For the ease of computation, only the compartments corresponding to infected and asymptomatically infected compartments are considered [20]. First, the original nonlinear ODE system is decomposed into two column vectors $\mathscr{F}=\left(\mathscr{F}_{i}\right)$ and $\mathscr{V}=\left(\mathscr{V}_{i}\right)$, where $\mathscr{F}_{i}$ is the $i^{\text {th }}$ row of $\mathscr{F}$ representing the rate at which new infections appear in compartment $i$, and $\mathscr{V}_{i}$ is the $i^{\text {th }}$ row of $\mathscr{V}$. Moreover, $\mathscr{V}_{i}=\mathscr{V}_{i}^{-}-\mathscr{V}_{i}^{+}$, where $\mathscr{V}_{i}^{-}$represents the rate at which individuals transfer out of compartment $i$, and $\mathscr{V}_{i}^{+}$is the rate at which individuals transfer into compartment $i$ [23]. Assume that the number of infected and asymptomatically infected compartments is $m$. The Jacobian matrices $F$ denoting transmission, and $V$ denoting transition [20] are defined as:

$$
F=\left[\frac{\partial \mathscr{F}_{i}\left(x^{0}\right)}{\partial x_{j}}\right], \quad V=\left[\frac{\partial \mathscr{V}_{i}\left(x^{0}\right)}{\partial x_{j}}\right]
$$

where $x^{0}$ represents the disease free equilibrium (DFE), and $x_{j}$ is the number or proportion of infected individuals in compartment $j, j=1,2, \cdots, m$.

The spectral radius of a matrix $A$ is denoted by $\rho(A)$. The reproduction number, $R_{0}$, is defined as $\rho\left(F V^{-1}\right)$ [21]. To understand entries of $F V^{-1}$, called the next generation matrix, consider the consequence of an infected individual introduced into compartment $k$ in a population at DFE [23]. The $(i, j)$ entry of $F$ represents the rate at which new infected individuals in compartment $i$ are produced by infected individuals in compartment $j$ [23]. The $(j, k)$ entry of $V^{-1}$ represents the average time that an infected individual stays in compartment $j[23]$. Hence, the $(i, k)$ entry of $F V^{-1}$ represents the expected number of new infections in compartment $i$ resulting from the infected individual originally introduced into compartment $k$ [23], where $i, k=1,2, \cdots, m$. Note that matrix $F$ is nonnegative and $V$ is proved to be a nonsingular M-matrix [23]. Recall that an $n \times n$ matrix $A$ is an M-matrix if it can be expressed in the form $A=s I-B$, such that matrix $B$ is non-negative, and $s \geqslant \rho(B)[28]$. 
Next, we illustrate computational procedures for finding $R_{0}$ using the next generation matrix method for susceptible-exposed-infectious-recovered (SEIR) compartmental models, assuming a disease is transmittable within a species and between different species, and movement rates for all species are independent of disease status. Daily time steps are used in all models.

\subsection{Models for two species in two nodes}

We present two applications of a simplified system for a disease involving two species in a two-node network with movement between the two nodes. In the first example, $R_{0}$ is computed while assuming only horizontal transmission is taking place. In the second example, the first model is extended by introducing vertical transmission into one species. The reproduction number is then computed.

\subsection{1. $R_{0}$ for two species with only horizontal transmission}

Below, a compartmental model for an infectious disease incorporating four compartments $(J=$ $S, E, I, R)$, two species $(k=1,2)$, two nodes $(i=1,2)$, and only horizontal transmission is presented. The differential equations representing the dynamic behavior are:

$$
\begin{aligned}
\frac{d S_{k i}}{d t} & =r_{k i}-\beta_{1 k i} S_{k i} I_{1 i} / N_{1 i}-\beta_{2 k i} S_{k i} I_{2 i} / N_{2 i}-d_{k i} S_{k i}+\sum_{j=1, j \neq i}^{2} \omega_{k j i} S_{k j}-\sum_{j=1, j \neq i}^{2} \omega_{k i j} S_{k i} \\
\frac{d E_{k i}}{d t} & =\beta_{1 k i} S_{k i} I_{1 i} / N_{1 i}+\beta_{2 k i} S_{k i} I_{2 i} / N_{2 i}-\varepsilon_{k i} E_{k i}-d_{k i} E_{k i}+\sum_{j=1, j \neq i}^{2} \omega_{k j i} E_{k j}-\sum_{j=1, j \neq i}^{2} \omega_{k i j} E_{k i} \\
\frac{\mathrm{d} I_{k i}}{\mathrm{~d} t} & =\varepsilon_{k i} E_{k i}-\gamma_{k i} I_{k i}-d_{k i} I_{k i}+\sum_{j=1, j \neq i}^{2} \omega_{k j i} I_{k j}-\sum_{j=1, j \neq i}^{2} \omega_{k i j} I_{k i} \\
\frac{\mathrm{d} R_{k i}}{\mathrm{~d} t} & =\gamma_{k i} I_{k i}-d_{k i} R_{k i}+\sum_{j=1, j \neq i}^{2} \omega_{k j i} R_{k j}-\sum_{j=1, j \neq i}^{2} \omega_{k i j} R_{k i} .
\end{aligned}
$$

The number of newborn individuals of species $k$ in node $i$ per day is denoted by $r_{k i}$. The number of species $k$ individuals in node $i$ of compartment $J$ is denoted by $J_{k i}$, and the total number of species $k$ individuals in node $i$ is $N_{k i}=S_{k i}+E_{k i}+I_{k i}+R_{k i}$. Total individuals of species $k$ infected daily in node $i$ by species 1 and species 2 are $\beta_{1 k i} S_{k i} I_{1 i} / N_{1 i}$ and $\beta_{2 k i} S_{k i} I_{2 i} / N_{2 i}$, respectively. The number of deaths from each compartment $J$ per day is $d_{k i} J_{k i}$. After the incubation period, $\varepsilon_{k i} E_{k i}$ individuals transfer to infected compartment daily. Following the infection period, $\gamma_{k i} I_{k i}$ recover from the infection each day. Movement rates for species $k$ individuals in compartment $J$ in and out of node $i$ are $\sum_{j=1, j \neq i}^{2} \omega_{k j i} J_{k j}$ and $\sum_{j=1, j \neq i}^{2} \omega_{k i j} J_{k i}$, respectively.

Species $k$ quantity in compartment $J$ and the total number in node $i$ at DFE are denoted by $J_{k i}^{0}$ and $N_{k i}^{0}$, respectively. To compute $R_{0}$ using the next generation matrix method, we need to prove the existence and uniqueness of DFE. At DFE, $S_{1 i}^{0}=N_{1 i}^{0}$, and $S_{2 i}^{0}=N_{2 i}^{0}$, as $E_{1 i}^{0}=I_{1 i}^{0}=R_{1 i}^{0}=E_{2 i}^{0}=$ $I_{2 i}^{0}=R_{2 i}^{0}=0$. This is a special case of the model for Theorem 5 (see appendix), which determines the existence of a unique solution $\left[\begin{array}{ll}N_{1 i}^{0} & N_{2 i}^{0}\end{array}\right]^{T}$.

The equations related to exposed and infected compartments are ordered:

$$
\frac{d}{d t}\left[\begin{array}{llllllll}
E_{11} & E_{12} & E_{21} & E_{22} & I_{11} & I_{12} & I_{21} & I_{22}
\end{array}\right]^{T}=\mathscr{F}_{H}-\mathscr{V}_{H}, \text { where }
$$




$$
\mathscr{F}_{H}=\left[\begin{array}{c}
\beta_{211} S_{11} I_{21} / N_{21}+\beta_{111} S_{11} I_{11} / N_{11} \\
\beta_{212} S_{12} I_{22} / N_{22}+\beta_{112} S_{12} I_{12} / N_{12} \\
\beta_{121} S_{21} I_{11} / N_{11}+\beta_{221} S_{21} I_{21} / N_{21} \\
\beta_{122} S_{22} I_{12} / N_{12}+\beta_{222} S_{22} I_{22} / N_{22} \\
0 \\
0 \\
0 \\
0
\end{array}\right], \quad \mathscr{V}_{H}=\left[\begin{array}{c}
d_{11} E_{11}+\varepsilon_{11} E_{11}+\omega_{112} E_{11}-\omega_{121} E_{12} \\
d_{12} E_{12}+\varepsilon_{12} E_{12}+\omega_{121} E_{12}-\omega_{112} E_{11} \\
d_{21} E_{21}+\varepsilon_{21} E_{21}+\omega_{212} E_{21}-\omega_{221} E_{22} \\
d_{22} E_{22}+\varepsilon_{22} E_{22}+\omega_{221} E_{22}-\omega_{212} E_{21} \\
-\varepsilon_{11} E_{11}+d_{11} I_{11}+\gamma_{11} I_{11}+\omega_{112} I_{11}-\omega_{121} I_{12} \\
-\varepsilon_{12} E_{12}+d_{12} I_{12}+\gamma_{12} I_{12}+\omega_{121} I_{12}-\omega_{112} I_{11} \\
-\varepsilon_{21} E_{21}+d_{21} I_{21}+\gamma_{21} I_{21}+\omega_{212} I_{21}-\omega_{221} I_{22} \\
-\varepsilon_{22} E_{22}+d_{22} I_{22}+\gamma_{22} I_{22}+\omega_{221} I_{22}-\omega_{212} I_{21}
\end{array}\right] .
$$

By (1), the Jacobian matrices for this model are:

$$
F_{H}=\left[\begin{array}{cc}
0_{4 \times 4} & \mathcal{A} \\
0 & 0_{4 \times 4}
\end{array}\right], \quad V_{H}=\left[\begin{array}{cc}
\oplus_{k=1}^{2} M_{k} & 0 \\
-\oplus_{k=1}^{2}\left(\oplus_{i=1}^{2} \varepsilon_{k i}\right) & \oplus_{k=1}^{2} X_{k}
\end{array}\right]
$$

where the symbol $\bigoplus$ represents the direct sum of matrices, i.e., $A \oplus B=\left[\begin{array}{cc}A & 0 \\ 0 & B\end{array}\right]$ for matrices $A$ and $B$. The subscript of the zero blocks, $4 \times 4$, indicates the size of the block. Matrices $\mathcal{A}, M_{k}$ and $X_{k}$ are:

$$
\begin{gathered}
\mathcal{A}=\left[\begin{array}{cccc}
\beta_{111} \frac{S_{11}^{0}}{N_{11}^{0}} & 0 & \beta_{211} \frac{S_{11}^{0}}{N_{21}^{0}} & 0 \\
0 & \beta_{112} \frac{S_{12}^{0}}{N_{12}^{0}} & 0 & \beta_{212} \frac{S_{12}^{0}}{N_{22}^{0}} \\
\beta_{121} \frac{S_{21}^{0}}{N_{11}^{0}} & 0 & \beta_{221} \frac{S_{21}^{0}}{N_{21}^{0}} & 0 \\
0 & \beta_{122} \frac{S_{22}^{0}}{N_{12}^{0}} & 0 & \beta_{222} \frac{S_{22}^{0}}{N_{22}^{0}}
\end{array}\right], \\
M_{1}=\left[\begin{array}{cc}
d_{11}+\varepsilon_{11}+\omega_{112} & -\omega_{121} \\
-\omega_{112} & d_{12}+\varepsilon_{12}+\omega_{121}
\end{array}\right], M_{2}=\left[\begin{array}{cc}
d_{21}+\varepsilon_{21}+\omega_{212} & -\omega_{221} \\
-\omega_{212} & d_{22}+\varepsilon_{22}+\omega_{221}
\end{array}\right], \\
X_{1}=\left[\begin{array}{cc}
d_{11}+\gamma_{11}+\omega_{112} & -\omega_{121} \\
-\omega_{112} & d_{12}+\gamma_{12}+\omega_{121}
\end{array}\right], X_{2}=\left[\begin{array}{cc}
d_{21}+\gamma_{21}+\omega_{212} & -\omega_{221} \\
-\omega_{212} & d_{22}+\gamma_{22}+\omega_{221}
\end{array}\right] .
\end{gathered}
$$

Because the matrices $M_{1}, M_{2}, X_{1}$, and $X_{2}$ are all invertible, we can readily check:

$$
V_{H}^{-1}=\left[\begin{array}{cc}
\oplus_{k=1}^{2} M_{k}^{-1} & 0 \\
\oplus_{k=1}^{2} \mathcal{Z}_{k} & \oplus_{k=1}^{2} X_{k}^{-1}
\end{array}\right]
$$

where $\mathcal{Z}_{k}=X_{k}^{-1}\left(\oplus_{i=1}^{2} \varepsilon_{k i}\right) M_{k}^{-1}$. The spectral radius of the next generation matrix $F_{H} V_{H}^{-1}$ is:

$$
\rho\left(F_{H} V_{H}^{-1}\right)=\rho\left(\left[\begin{array}{cc}
0_{4 \times 4} & \mathcal{A} \\
0 & 0_{4 \times 4}
\end{array}\right]\left[\begin{array}{cc}
\oplus_{k=1}^{2} M_{k}^{-1} & 0 \\
\oplus_{k=1}^{2} \mathcal{Z}_{k} & \oplus_{k=1}^{2} X_{k}^{-1}
\end{array}\right]\right)=\rho\left(\mathcal{A}\left(\oplus_{k=1}^{2} \mathcal{Z}_{k}\right)\right) .
$$

Therefore,

$$
R_{0}^{H}:=\rho\left(F_{H} V_{H}^{-1}\right)=\rho\left(\mathcal{A}\left(\oplus_{k=1}^{2} \mathcal{Z}_{k}\right)\right)
$$

where $R_{0}^{H}$ is the reproduction number for horizontal transmission.

\subsection{2. $R_{0}$ for two species with vertical transmission in one species}

We keep the model for species 2 (Equation (2) to (5) with $k=2$ ), while extending the model for species 1 by incorporating vertical transmission. The model for species 1 is:

$$
\begin{aligned}
& \frac{d P_{1 i}}{d t}=r_{1 i}-b_{1} q_{1 i} I_{1 i}-\theta_{1 i} P_{1 i} \\
& \frac{d Q_{1 i}}{d t}=b_{1 i} q_{1 i} I_{1 i}-\theta_{1 i} Q_{1 i}
\end{aligned}
$$




$$
\begin{aligned}
& \frac{d S_{1 i}}{d t}=\theta_{1 i} P_{1 i}-\beta_{11 i} S_{1 i} I_{1 i} / N_{1 i}-\beta_{21 i} S_{1 i} I_{2 i} / N_{2 i}-d_{1 i} S_{1 i}+\sum_{j=1, j \neq i}^{2} \omega_{1 j i} S_{1 j}-\sum_{j=1, j \neq i}^{2} \omega_{1 i j} S_{1 i} \\
& \frac{d E_{1 i}}{d t}=\beta_{11 i} S_{1 i} I_{1 i} / N_{1 i}+\beta_{21 i} S_{1 i} I_{2 i} / N_{2 i}-\varepsilon_{1 i} E_{1 i}-d_{1 i} E_{1 i}+\sum_{j=1, j \neq i}^{2} \omega_{1 j i} E_{1 j}-\sum_{j=1, j \neq i}^{2} \omega_{1 i j} E_{1 i} \\
& \frac{\mathrm{d} I_{1 i}}{\mathrm{~d} t}=\theta_{1 i} Q_{1 i}+\varepsilon_{1 i} E_{1 i}-\gamma_{1 i} I_{1 i}-d_{1 i} I_{1 i}+\sum_{j=1, j \neq i}^{2} \omega_{1 j i} I_{1 j}-\sum_{j=1, j \neq i}^{2} \omega_{1 i j} I_{1 i} \\
& \frac{\mathrm{d} R_{1 i}}{\mathrm{~d} t}=\gamma_{1 i} I_{1 i}-d_{1 i} R_{1 i}+\sum_{j=1, j \neq i}^{2} \omega_{1 j i} R_{1 j}-\sum_{j=1, j \neq i}^{2} \omega_{1 i j} R_{1 i} .
\end{aligned}
$$

The number of eggs laid by species 1 per day is denoted as $r_{1 i}$, including $b_{1 i} q_{1 i} I_{1 i}$ infected eggs, and $r_{1 i}-b_{1 i} q_{1 i} I_{1 i}$ uninfected eggs. After the development period, $\theta_{1 i} P_{1 i}$ eggs develop into susceptible adults, and $\theta_{1 i} Q_{1 i}$ eggs develop into infected adults daily. The interpretations of other terms are the same as corresponding terms described in Section 2.1.1.

At DFE, $Q_{1 i}^{0}=E_{1 i}^{0}=I_{1 i}^{0}=R_{1 i}^{0}=E_{2 i}^{0}=I_{2 i}^{0}=R_{2 i}^{0}=0, S_{1 i}^{0}=N_{1 i}^{0}$, and $S_{2 i}^{0}=N_{2 i}^{0}$. Since this is another special case of the model for Theorem 5 , a unique solution $\left[\begin{array}{ll}N_{1 i}^{0} & N_{2 i}^{0}\end{array}\right]^{T}$ exists. In our second model, the equations related to exposed and infected compartments are ordered:

$$
\begin{aligned}
& \frac{d}{d t}\left[\begin{array}{llllllllll}
Q_{11} & Q_{12} & E_{11} & E_{12} & E_{21} & E_{22} & I_{11} & I_{12} & I_{21} & I_{22}
\end{array}\right]^{T}=\mathscr{F}-\mathscr{V} \text {, where } \\
& \mathscr{F}=\left[\begin{array}{c}
b_{11} q_{11} I_{11} \\
b_{12} q_{12} I_{12} \\
\beta_{211} S_{11} I_{21} / N_{21}+\beta_{111} S_{11} I_{11} / N_{11} \\
\beta_{212} S_{12} I_{22} / N_{22}+\beta_{112} S_{12} I_{12} / N_{12} \\
\beta_{121} S_{21} I_{11} / N_{11}+\beta_{221} S_{21} I_{21} / N_{21} \\
\beta_{122} S_{22} I_{12} / N_{12}+\beta_{222} S_{22} I_{22} / N_{22} \\
0 \\
0 \\
0 \\
0
\end{array}\right], \mathscr{V}=\left[\begin{array}{c}
\theta_{11} Q_{11} \\
\theta_{12} Q_{12} \\
d_{11} E_{11}+\varepsilon_{11} E_{11}+\omega_{112} E_{11}-\omega_{121} E_{12} \\
d_{12} E_{12}+\varepsilon_{12} E_{12}+\omega_{121} E_{12}-\omega_{112} E_{11} \\
d_{21} E_{21}+\varepsilon_{21} E_{21}+\omega_{212} E_{21}-\omega_{221} E_{22} \\
d_{22} E_{22}+\varepsilon_{22} E_{22}+\omega_{221} E_{22}-\omega_{212} E_{21} \\
-\theta_{11} Q_{11}-\varepsilon_{11} E_{11}+d_{11} I_{11}+\gamma_{11} I_{11}+\omega_{112} I_{11}-\omega_{121} I_{12} \\
-\theta_{12} Q_{12}-\varepsilon_{12} E_{12}+d_{12} I_{12}+\gamma_{12} I_{12}+\omega_{121} I_{12}-\omega_{112} I_{11} \\
-\varepsilon_{21} E_{21}+d_{21} I_{21}+\gamma_{21} I_{21}+\omega_{212} I_{21}-\omega_{221} I_{22} \\
-\varepsilon_{22} E_{22}+d_{22} I_{22}+\gamma_{22} I_{22}+\omega_{221} I_{22}-\omega_{212} I_{21}
\end{array}\right] .
\end{aligned}
$$

By (1), the Jacobian matrices for this model are:

$$
F=\left[\begin{array}{cc}
0_{2 \times 2} & U_{2 \times 8} \\
0_{8 \times 2} & F_{H}
\end{array}\right], \quad V=\left[\begin{array}{cc}
\oplus_{i=1}^{2} \theta_{1 i} & 0_{2 \times 8} \\
W_{8 \times 2} & V_{H}
\end{array}\right] .
$$

Here $F_{H}$ and $V_{H}$ are the matrices in (6) and

$$
U=\left[\begin{array}{lll}
0_{2 \times 4} & \bigoplus_{i=1}^{2} b_{1 i} q_{1 i} & 0_{2 \times 2}
\end{array}\right], \quad W=\left[\begin{array}{c}
0_{4 \times 2} \\
-\bigoplus_{i=1}^{2} \theta_{1 i} \\
0_{2 \times 2}
\end{array}\right] .
$$

The matrix $V^{-1}$ and the next generation matrix $F V^{-1}$ are:

$$
V^{-1}=\left[\begin{array}{cc}
\bigoplus_{i=1}^{2} \theta_{1 i}^{-1} & 0 \\
-V_{H}^{-1} W\left(\bigoplus_{i=1}^{2} \theta_{1 i}^{-1}\right) & V_{H}^{-1}
\end{array}\right], \quad F V^{-1}=\left[\begin{array}{cc}
-U V_{H}^{-1} W\left(\bigoplus_{i=1}^{2} \theta_{1 i}^{-1}\right) & U V_{H}^{-1} \\
-F_{H} V_{H}^{-1} W\left(\bigoplus_{i=1}^{2} \theta_{1 i}^{-1}\right) & F_{H} V_{H}^{-1}
\end{array}\right] .
$$

Since $\mathcal{M}^{-1}\left(F V^{-1}\right) \mathcal{M}=\left[\begin{array}{cc}0 & U V_{H}^{-1} \\ 0 & F_{H} V_{H}^{-1}-W\left(\bigoplus_{i=1}^{2} \theta_{1 i}^{-1}\right) U V_{H}^{-1}\end{array}\right]$, where $\mathcal{M}=\left[\begin{array}{cc}I_{2 \times 2} & 0 \\ W\left(\bigoplus_{i=1}^{2} \theta_{1 i}^{-1}\right) & I_{8 \times 8}\end{array}\right]$, we have

$$
R_{0}=\rho\left(F V^{-1}\right)=\rho\left(F_{H} V_{H}^{-1}-W\left(\bigoplus_{i=1}^{2} \theta_{1 i}^{-1}\right) U V_{H}^{-1}\right) .
$$


$R_{0}$ is a function of vertical and horizontal transmission parameters. Since $F_{H} V_{H}^{-1}$ and $-W\left(\bigoplus_{i=1}^{2} \theta_{1 i}^{-1}\right) U V_{H}^{-1}$ are both nonnegative matrices, by Theorem 4 in appendix,

$$
R_{0} \geqslant \rho\left(F_{H} V_{H}^{-1}\right) .
$$

\section{2. $R_{0}$ for multiple species in a general network}

The model presented in Section 2.1.2 is generalized to model diseases transmitted among all $h$ species in node $i(i=1,2, \cdots, n)$. Suppose a disease is transmitted by species $k(k=1,2, \cdots, h)$ vertically and horizontally if $1 \leqslant k \leqslant g$ and only horizontally otherwise. The dynamical behavior is given by the system with $4 h n+2 g n$ differential equations:

$$
\begin{aligned}
\frac{d P_{k i}}{d t} & =\left[r_{k i}-b_{k i} q_{k i} I_{k i}-\theta_{k i} P_{k i}\right] \delta(k) \\
\frac{d Q_{k i}}{d t} & =\left[b_{k i} q_{k i} I_{k i}-\theta_{k i} Q_{k i}\right] \delta(k) \\
\frac{d S_{k i}}{d t} & =\theta_{k i} P_{k i} \delta(k)+r_{k i}(1-\delta(k))-\sum_{m=1}^{h} \beta_{m k i} S_{k i} I_{m i} / N_{m i}-d_{k i} S_{k i}+\sum_{j=1, j \neq i}^{n} \omega_{k j i} S_{k j}-\sum_{j=1, j \neq i}^{n} \omega_{k i j} S_{k i} \\
\frac{d E_{k i}}{d t} & =\sum_{m=1}^{h} \beta_{m k i} S_{k i} I_{m i} / N_{m i}-\varepsilon_{k i} E_{k i}-d_{k i} E_{k i}+\sum_{j=1, j \neq i}^{n} \omega_{k j i} E_{k j}-\sum_{j=1, j \neq i}^{n} \omega_{k i j} E_{k i} \\
\frac{\mathrm{d} I_{k i}}{\mathrm{~d} t} & =\theta_{k i} Q_{k i} \delta(k)+\varepsilon_{k i} E_{k i}-\gamma_{k i} I_{k i}-d_{k i} I_{k i}+\sum_{j=1, j \neq i}^{n} \omega_{k j i} I_{k j}-\sum_{j=1, j \neq i}^{n} \omega_{k i j} I_{k i} \\
\frac{\mathrm{d} R_{k i}}{\mathrm{~d} t} & =\gamma_{k i} I_{k i}-d_{k i} R_{k i}+\sum_{j=1, j \neq i}^{n} \omega_{k j i} R_{k j}-\sum_{j=1, j \neq i}^{n} \omega_{k i j} R_{k i} .
\end{aligned}
$$

The daily number of species $k$ individuals infected by species $m$ is $\beta_{m k i} S_{k i} I_{m i} / N_{m i}$. The daily numbers of species $k$ individuals in compartment $J$ moving in and out of node $i$ are $\sum_{j=1, j \neq i}^{n} \omega_{k j i} J_{k j}$ and $\sum_{j=1, j \neq i}^{n} \omega_{k i j} J_{k i}$, respectively. Other terms in the above equations have the same meanings as the corresponding ones in Section 2.1.1 (Equation (2) to (5)) and Section 2.1.2 (Equation (10) to (15)) except $\delta(k)$ defined below, which is used to differentiate the horizontally-transmitting species and the species exhibiting both types of transmission.

$$
\delta(k)= \begin{cases}1 & \text { for } \quad 1 \leq k \leq g \\ 0 & \text { for } \quad g+1 \leq k \leq h .\end{cases}
$$

To compute $R_{0}$ using the next generation matrix method, we need to find matrices $\mathscr{F}$ and $\mathscr{V}$, omitted here due to large size. In determining Jacobian matrices $F$ and $V$, the infected variables are ordered by compartment, species, and node index, i.e.,

$$
\begin{aligned}
& Q_{11}, Q_{12}, \cdots, Q_{1 n}, Q_{21}, Q_{22}, \cdots Q_{2 n}, \cdots, Q_{g 1}, Q_{g 2}, \cdots, Q_{g n}, \\
& E_{11}, E_{12}, \cdots, E_{1 n}, E_{21}, E_{22}, \cdots, E_{2 n}, \cdots, E_{h 1}, E_{h 2}, \cdots, E_{h n}, \\
& I_{11}, I_{12}, \cdots, I_{1 n}, I_{21}, I_{22}, \cdots, I_{2 n}, \cdots, I_{h 1}, I_{h 2}, \cdots, I_{h n} .
\end{aligned}
$$

At DFE, $Q_{k i}=E_{k i}=I_{k i}=R_{k i}=0$, and $S_{k i}=N_{k i}$. By Theorem 5 in appendix, a unique solution $\left[\begin{array}{ll}N_{k 1}^{0} & N_{k 2}^{0} \cdots N_{k n}^{0}\end{array}\right]^{T}$ exists. Since incorporating multiple species in multiple nodes leads to matrices $F$ and $V$ growing very large, the computation of $R_{0}$ is simplified by decomposing the matrices into blocks, deriving block upper or lower triangular matrices as follows:

$$
F=\left[\begin{array}{cc}
0_{g n \times g n} & U_{g n \times 2 h n} \\
0_{2 h n \times g n} & F_{H}
\end{array}\right], \quad V=\left[\begin{array}{cc}
\bigoplus_{k=1}^{g}\left(\bigoplus_{i=1}^{n} \theta_{k i}\right) & 0_{g n \times 2 h n} \\
W_{2 h n \times g n} & V_{H}
\end{array}\right],
$$


where

$$
\begin{gathered}
F_{H}=\left[\begin{array}{ll}
0_{h n \times h n} & \mathcal{A}_{h n \times h n} \\
0_{h n \times h n} & 0_{h n \times h n}
\end{array}\right], \quad V_{H}=\left[\begin{array}{cc}
\bigoplus_{k=1}^{h} M_{k} & 0_{h n \times h n} \\
-\bigoplus_{k=1}^{h}\left(\bigoplus_{i=1}^{n} \varepsilon_{k i}\right) & \bigoplus_{k=1}^{h} X_{k}
\end{array}\right], \\
U=\left[\begin{array}{lll}
0_{g n \times h n} & \bigoplus_{k=1}^{g}\left(\bigoplus_{i=1}^{n} b_{k i} q_{k i}\right) & 0_{g n \times(h-g) n}
\end{array}\right], \quad W=\left[\begin{array}{c}
0_{h n \times g n} \\
-\bigoplus_{k=1}^{g}\left(\bigoplus_{i=1}^{n} \theta_{k i}\right) \\
0_{(h-g) n \times g n}
\end{array}\right] .
\end{gathered}
$$

The block matrix $\mathcal{A}$ in $F_{H}$ is written into an $h \times h$ block matrix $\mathcal{A}=\left(\mathcal{A}_{k m}\right)$ and its $(k, m)$ entry is an $n \times n$ diagonal matrix $\mathcal{A}_{k m}=\bigoplus_{i=1}^{n}\left(\beta_{m k i} \frac{S_{k i}^{0}}{N_{m i}^{0}}\right)$. The matrices $M_{k}$ and $X_{k}$ are:

$$
M_{k}=\left[\begin{array}{cccc}
\zeta_{k 1} & -\omega_{k 21} & \cdots & -\omega_{k n 1} \\
-\omega_{k 12} & \zeta_{k 2} & \cdots & -\omega_{k n 2} \\
\cdots & \cdots & \cdots & \cdots \\
-\omega_{k 1 n} & \cdots & \cdots & \zeta_{k n}
\end{array}\right], \quad \text { and } \quad X_{k}=M_{k}+\bigoplus_{i=1}^{n}\left(\gamma_{k i}-\varepsilon_{k i}\right) \text {, }
$$

where $\zeta_{k i}=d_{k i}+\varepsilon_{k i}+\sum_{j=1, j \neq i}^{n} \omega_{k i j}$. Since matrices $M_{k}$ and $X_{k}$ are invertible, according to Theorem 7, $V_{H}$ and $V$ are invertible. It is easy to check:

$$
V_{H}^{-1}=\left[\begin{array}{cc}
\oplus_{k=1}^{h} M_{k}^{-1} & 0 \\
\oplus_{k=1}^{h} \mathcal{Z}_{k} & \oplus_{k=1}^{h} X_{k}^{-1}
\end{array}\right], \quad V^{-1}=\left[\begin{array}{cc}
\bigoplus_{k=1}^{g}\left(\bigoplus_{i=1}^{n} \theta_{k i}^{-1}\right) & 0_{g n \times 2 h n} \\
-V_{H}^{-1} W\left(\bigoplus_{k=1}^{g}\left(\bigoplus_{i=1}^{n} \theta_{k i}^{-1}\right)\right) & V_{H}^{-1}
\end{array}\right],
$$

where $\mathcal{Z}_{k}=X_{k}^{-1}\left(\oplus_{i=1}^{n} \varepsilon_{k i}\right) M_{k}^{-1}$. Similar to the derivation in Section 2.1.2, $R_{0}$ is:

$$
R_{0}=\rho\left(F V^{-1}\right)=\rho\left(F_{H} V_{H}^{-1}-W\left(\bigoplus_{k=1}^{g}\left(\bigoplus_{i=1}^{n} \theta_{k i}^{-1}\right)\right) U V_{H}^{-1}\right) .
$$

Moreover, (17) still holds. If the lower bound $\rho\left(F_{H} V_{H}^{-1}\right)>1$, we can conclude that a network may be invaded without computing the upper bound or the exact value of $R_{0}$.

The term $F_{H} V_{H}^{-1}$ is related to horizontal transmission, and the term $-W\left(\bigoplus_{k=1}^{g}\left(\bigoplus_{i=1}^{n} \theta_{k i}^{-1}\right)\right) U V_{H}^{-1}$ is related to vertical transmission, making $R_{0}$ a function of vertical and horizontal transmission parameters. Generally, $R_{0}$ depends on demographic, disease and movement factors, proving too complicated to compute or analyze [7]. The complexity of computing $R_{0}$ using Equation (26) depends on a specific model for a certain disease. For the general model, we can only provide the formula of $R_{0}$ in Equation (26) and its lower bound in Inequality (17).

In the following section, Equation (26) is applied to an RVF virus transmission meta-population model. Then, based on the assumptions for the RVF model, we compute $R_{0}$ using Equation (26) and further derive lower bound and upper bound, providing insights into the role of model parameters on $R_{0}$.

\section{The application of proposed method to RVF meta-population model}

Rift Valley fever is an emerging mosquito-borne disease mainly affecting and colonizing domestic ruminants and humans [29, 30]. Main vectors of RVF include Aedes and Culex mosquitoes [30]. Humans and ruminants are main hosts [30]. Aedes mosquitoes are believed to be initial source of RVF outbreaks [31], since RVF virus-carrying eggs can survive in drought area soil for many years, later breeding infected mosquitoes in flooded habitats [32,33]. Ruminants infected by mosquito bites [29] can transmit RVF virus to Aedes feeding on them as blood meals [30]. Culex mosquitoes also amplify RVF virus transmission by ingesting blood from infected ruminants [29]. Most humans acquire RVF virus infection when bitten by infected mosquitoes or during contact with body fluid of infected ruminants [34]. Next, we derive $R_{0}$ for an RVF meta-population model to study the role of parameters and networks on the reproduction number. 


\subsection{The network-based RVF meta-population model}

In this section, the general model in Equations (18) to (23) of Section 2.2 is applied to study the dynamics of RVF virus transmission with $h=4, g=1$. Aedes and Culex mosquito vectors are considered in the model, as are livestock and human hosts. The RVF model is less complex than the general model presented in Equations (18) to (23). Here, we assume only livestock can move in and out of nodes, and all mosquitoes do not recover. We consider disease-induced mortality for livestock and humans, and carrying capacity for mosquitoes and humans. Due to lack of transmission by humans or direct intra-species transmission, this RVF model contains fewer infection terms than those in the general model. See appendix for the full model (Equations (47) to (67)) and relative parameters (Table $2)$. The number of species $k$ individuals $(k=1,2,3,4)$ from node $i(i=1,2, \cdots, n)$ in compartment $J$ is represented by $J_{k i}$, where $k=1$ (resp. 2, 3,4) represents Aedes mosquitoes (resp. livestock, Culex mosquitoes, and humans). The parameter $r_{2 i}$ is the number of livestock born daily in node $i(i=1,2, \cdots, n)$. The daily numbers of new born Aedes mosquitoes, Culex mosquitoes, and humans are $b_{k i} N_{k i}$. A node index is added at the end of the subscript of a parameter only when referring to a parameter for a specific node. For example, $\beta_{12 i}$ represents the contact rate from Aedes mosquitoes $(k=1)$ to livestock $(k=2)$ in node $i$.

\subsection{The computation of $R_{0}$ for $R V F$}

The explicit expression of $R_{0}$ in Equation (26) is applied to the RVF meta-population model. The above assumptions allow us to obtain the lower and upper bounds of $R_{0}$.

\subsubsection{Explicit expression of $R_{0}$ for $R V F$}

First, we check if a unique solution $N_{k i}^{0}$ exists. At DFE, $E_{k i}^{0}=I_{k i}^{0}=R_{k i}^{0}=0$. By computation, $S_{k i}^{0}=N_{k i}^{0}=\frac{b_{k i} K_{k}}{d_{k i}}$ for $k=1,3,4$, where $K_{k}$ is the carrying capacity of species $k$. This is a special case of the model for Theorem 5, which generates a unique nonnegative solution for the total number of livestock in node $i$ at DFE denoted by: $\left[\begin{array}{llll}N_{21}^{0} & N_{22}^{0} & \cdots & N_{2 n}^{0}\end{array}\right]^{T}$.

By (1), the Jacobian matrices for the RVF model are:

$$
F=\left[\begin{array}{cc}
0_{n \times n} & U_{n \times 8 n} \\
0_{8 n \times n} & F_{H}
\end{array}\right], \quad V=\left[\begin{array}{cc}
\oplus_{i=1}^{n} \theta_{1 i} & 0_{n \times 8 n} \\
W_{8 n \times n} & V_{H}
\end{array}\right] .
$$

Each component of the $R_{0}$ formula is computed as follows:

$$
\begin{gathered}
F_{H}=\left[\begin{array}{ll}
0_{4 n \times 4 n} & \mathcal{A}_{4 n \times 4 n} \\
0_{4 n \times 4 n} & 0_{4 n \times 4 n}
\end{array}\right], \quad V_{H}=\left[\begin{array}{cc}
\oplus_{k=1}^{4} M_{k} & 0_{4 n \times 4 n} \\
-\left(\oplus_{k=1}^{4}\left(\oplus_{i=1}^{n} \varepsilon_{k i}\right)\right)_{4 n \times 4 n} & \bigoplus_{k=1}^{4} X_{k}
\end{array}\right] . \\
U=\left[\begin{array}{lll}
0_{n \times 4 n} & \oplus_{i=1}^{n}\left(b_{1 i} q_{1 i}\right) & 0_{n \times 3 n}
\end{array}\right], \quad W=\left[\begin{array}{c}
0_{4 n \times n} \\
-\left(\oplus_{i=1}^{n} \theta_{1 i}\right) \\
0_{3 n \times n}
\end{array}\right] . \\
\mathcal{A}=\left[\begin{array}{cccc}
0 & \mathcal{A}_{12} & 0 & 0 \\
\mathcal{A}_{21} & 0 & \mathcal{A}_{23} & 0 \\
0 & \mathcal{A}_{32} & 0 & 0 \\
\mathcal{A}_{41} & \mathcal{A}_{42} & \mathcal{A}_{43} & 0
\end{array}\right], \\
\mathcal{A}_{12}=\oplus_{i=1}^{n} \beta_{21 i} \frac{S_{1 i}^{0}}{N_{2 i}^{0},} \quad \mathcal{A}_{21}=\oplus_{i=1}^{n} \beta_{12 i} \frac{S_{2 i}^{0}}{N_{1 i}^{0},} \quad \mathcal{A}_{23}=\oplus_{i=1}^{n} \beta_{32 i} \frac{S_{2 i}^{0}}{N_{3 i}^{0}}, \quad \mathcal{A}_{32}=\oplus_{i=1}^{n} \beta_{23 i} \frac{S_{3 i}^{0}}{N_{2 i}^{0}}, \\
\mathcal{A}_{41}=\oplus_{i=1}^{n} \beta_{14 i} \frac{S_{4 i}^{0}}{N_{1 i}^{0},}
\end{gathered}
$$


The matrices $V_{H}^{-1}$ and $V^{-1}$ are in Equation (25) with $\mathrm{g}=1$ and $\mathrm{h}=4$, respectively. Below, matrices $M_{k}$ and $X_{k}$ relate to Aedes mosquitoes, livestock, Culex mosquitoes, and humans with $k=1,2,3,4$, respectively.

$$
\begin{aligned}
& M_{1}=\oplus_{i=1}^{n}\left(\frac{d_{1 i} N_{1 i}^{0}}{K_{1}}+\varepsilon_{1 i}\right), \quad X_{1}=M_{1}-\oplus_{i=1}^{n} \varepsilon_{1 i}, \\
& M_{3}=\oplus_{i=1}^{n}\left(\frac{d_{3 i} N_{3 i}^{0}}{K_{3}}+\varepsilon_{3 i}\right), \quad X_{3}=M_{3}-\oplus_{i=1}^{n} \varepsilon_{3 i}, \\
& M_{4}=\oplus_{i=1}^{n}\left(\frac{d_{4 i} N_{4 i}^{0}}{K_{4}}+\varepsilon_{4 i}\right), \quad X_{4}=M_{4}-\oplus_{i=1}^{n} \varepsilon_{4 i}, \\
& M_{2}=\left[\begin{array}{cccc}
\zeta_{21} & -\omega_{221} & \cdots & -\omega_{2 n 1} \\
-\omega_{212} & \zeta_{22} & \cdots & -\omega_{2 n 2} \\
\cdots & \cdots & \cdots & \cdots \\
-\omega_{21 n} & -\omega_{22 n} & \cdots & \zeta_{2 n}
\end{array}\right], \quad X_{2}=M_{2}+\oplus_{i=1}^{n}\left(\gamma_{2 i}+\mu_{2 i}-\varepsilon_{2 i}\right) .
\end{aligned}
$$

The reproduction number, $R_{0}$ can be computed by plugging the above terms into Equation (26). Typically $R_{0}$ for a meta-population model is complicated [35]. Deriving some bounds on the value of $R_{0}$ can be helpful [35]. In the following section, we derive lower and upper bounds for $R_{0}$.

\subsubsection{Deriving lower bound and upper bound for $R_{0}$}

Bounds of $R_{0}$ are derived in many articles, among which are some following examples. Gao and Ruan present bounds of $R_{0}$ for an SIS patch model [36] investigating effects of media coverage and human movement on the spread of infectious diseases, as well as a malaria model [37]. Hsieh, Driessche, and Wang [38] derive bounds of $R_{0}$, describing the relationship between the reproduction numbers for the isolated $i^{t h}$ patch and for the system. Salmani and Driessche [1] derive bounds for an SEIRS patch model. Arino [35] presents bounds of $R_{0}$ for patch models considering multiple species. The reproduction number for an averaging process of mixed individuals or groups is estimated to be smaller than or equal to the reproduction number before mixing [39]. We derive bounds of $R_{0}$ for RVF metapopulation model in this section. In the following, we shall state main results and prove them in appendix.

Theorem 1. Consider the model presented in Section 3.1 (Equations (47) to (67)), we obtain

$$
\rho\left(F_{H} V_{H}^{-1}\right) \leqslant R_{0} \leqslant \rho\left(F_{H} V_{H}^{-1}\right)+\max _{i}\left(q_{1 i}\right) .
$$

The difference between the lower and upper bounds is $\max _{i}\left(q_{1 i}\right)$ with lower bound $\rho\left(F_{H} V_{H}^{-1}\right)$ computed by Equation (43).

Theorem 2. For the model in Section 3.1 (Equations (47) to (67)), assume $\varepsilon_{2 i}=\varepsilon_{2}$ for all $i$, then

$$
\sqrt{\min _{i}\left(\chi_{i}\right) \rho\left(X_{2}^{-1} M_{2}^{-1}\right)} \leqslant R_{0} \leqslant \sqrt{\max _{i}\left(\chi_{i}\right) \rho\left(X_{2}^{-1} M_{2}^{-1}\right)}+\max _{i}\left(q_{1 i}\right),
$$

where

$$
\chi_{i}=\frac{\varepsilon_{1 i} \varepsilon_{2} \beta_{12 i} \beta_{21 i}}{b_{1 i}\left(b_{1 i}+\varepsilon_{1 i}\right)}+\frac{\varepsilon_{2} \varepsilon_{3 i} \beta_{32 i} \beta_{23 i}}{b_{3 i}\left(b_{3 i}+\varepsilon_{3 i}\right)} .
$$

The difference between the lower bound and the upper bound in a network with heterogeneous corresponding parameters across nodes is larger than that in Inequality (30).

Corollary 1. Suppose for all $i$, birth and incubation rates in mosquitoes and livestock, contact rates between livestock and mosquitoes are homogeneous for different nodes, i.e.,

$$
b_{1 i}=b_{1}, b_{3 i}=b_{3}, \varepsilon_{1 i}=\varepsilon_{1}, \varepsilon_{2 i}=\varepsilon_{2}, \varepsilon_{3 i}=\varepsilon_{3}, \beta_{12 i}=\beta_{12}, \beta_{21 i}=\beta_{21}, \beta_{23 i}=\beta_{23}, \beta_{32 i}=\beta_{32} .
$$


Then

$$
\sqrt{\chi \rho\left(X_{2}^{-1} M_{2}^{-1}\right)} \leqslant R_{0} \leqslant \sqrt{\chi \rho\left(X_{2}^{-1} M_{2}^{-1}\right)}+\max _{i}\left(q_{1 i}\right)
$$

where

$$
\chi=\frac{\varepsilon_{1} \varepsilon_{2} \beta_{12} \beta_{21}}{b_{1}\left(b_{1}+\varepsilon_{1}\right)}+\frac{\varepsilon_{2} \varepsilon_{3} \beta_{32} \beta_{23}}{b_{3}\left(b_{3}+\varepsilon_{3}\right)} .
$$

Theorem 3. Under the condition of Theorem 2, $R_{0}$ can be estimated by the following inequality:

$$
\sqrt{\frac{\min _{i}\left(\chi_{i}\right)}{\max _{i}\left(d_{2 i}+\varepsilon_{2}\right) \max _{i}\left(d_{2 i}+\gamma_{2 i}+\mu_{2 i}\right)}} \leqslant R_{0} \leqslant \sqrt{\frac{\max _{i}\left(\chi_{i}\right)}{\min _{i}\left(d_{2 i}+\varepsilon_{2}\right) \min _{i}\left(d_{2 i}+\gamma_{2 i}+\mu_{2 i}\right)}}+\max _{i}\left(q_{1 i}\right) .
$$

If the differences between $\min _{i}\left(\chi_{i}\right)$ and $\max _{i}\left(\chi_{i}\right), \min _{i}\left(d_{2 i}+\varepsilon_{2}\right)$ and $\max _{i}\left(d_{2 i}+\varepsilon_{2}\right), \min _{i}\left(d_{2 i}+\right.$ $\left.\gamma_{2 i}+\mu_{2 i}\right)$ and $\max _{i}\left(d_{2 i}+\gamma_{2 i}+\mu_{2 i}\right)$ are large, then the difference between the lower bound and the upper bound may be large. However, the scalar lower bound and upper bound are easily computed. Moreover, if the lower bound is greater than 1, we can conclude that the network may be invaded without computing $R_{0}$ or its upper bound.

Corollary 2. Based on the condition of Corollary 1, we further assume that for all $i$, the death rate, mortality rate, and recovery rate in livestock, and transovarial transmission rate in Aedes mosquitoes are homogeneous for all nodes, i.e.,

$$
d_{2 i}=d_{2}, \quad \mu_{2 i}=\mu_{2}, \quad \gamma_{2 i}=\gamma_{2}, \quad q_{1 i}=q_{1} .
$$

Then

$$
\sqrt{\frac{\chi}{\left(d_{2}+\varepsilon_{2}\right)\left(d_{2}+\gamma_{2}+\mu_{2}\right)}} \leqslant R_{0} \leqslant \sqrt{\frac{\chi}{\left(d_{2}+\varepsilon_{2}\right)\left(d_{2}+\gamma_{2}+\mu_{2}\right)}}+q_{1} .
$$

In this case, the lower and upper bounds of $R_{0}$ correspond to the bounds for homogeneous populations presented in [26] and are tight [26]. Clearly, $R_{0}$ for horizontal transmission,

$$
R_{0}^{H}=\sqrt{\frac{\varepsilon_{2}}{\left(b_{2}+\varepsilon_{2}\right)\left(b_{2}+\gamma_{2}+\mu_{2}\right)}\left[\frac{\varepsilon_{1} \beta_{12} \beta_{21}}{b_{1}\left(b_{1}+\varepsilon_{1}\right)}+\frac{\varepsilon_{3} \beta_{32} \beta_{23}}{b_{3}\left(b_{3}+\varepsilon_{3}\right)}\right]}
$$

does not depend on livestock movement rates. Only bounds for $R_{0}$ can theoretically be obtained. Based on numerical simulation results, we conjecture that, given the conditions for Corollary $2, R_{0}$ does not depend on livestock movement rates.

\subsubsection{Tightness of bounds for $R_{0}$}

A one hundred-node network with heterogeneous corresponding parameters among nodes is built to study the tightness of bounds. We uniformly distribute disease parameters for each node during one hundred runs within their respective ranges, given in Table 2 . Then, $R_{0}$ is numerically computed according to Equation (26). Lower and upper bounds of $R_{0}$ are computed according to Inequality (30) in Theorem 1. The reproduction number for horizontal transmission is computed according to Equation (43). The lower bound of $R_{0}$ (denoted by $R_{0}^{L}$ ) versus $R_{0}$ in each run is shown in Figure 1(a), and the upper bound of $R_{0}$ (denoted by $R_{0}^{U}$ ) versus $R_{0}$ in each run is shown in Figure 1(b). In each run, the upper bound is slightly greater and the lower bound is slightly smaller than $R_{0}$. With the same network and the same set of parameters, the lower and upper bounds of $R_{0}$ are computed using Inequality (31). The lower bound versus exact $R_{0}$ is shown in Figure $2(a)$, and the upper bound versus exact $R_{0}$ is shown in Figure 2(b). The bounds obtained by Inequality (31) in Theorem 2 are less tight 
than those obtained by Inequality (30) in Theorem 1 , as $\rho\left(F_{H} V_{H}^{-1}\right)$ is estimated by computing the spectral radius of a smaller size matrix. The bounds obtained by Inequality (36) in Theorem 3 can be even looser because $\rho\left(X_{2}^{-1} M_{2}^{-1}\right)$ is simply estimated by scalars.

The above bounds are for heterogeneous networks. The bounds in Corollary 2 (see Inequality (38)) apply to homogeneous networks, where the difference between the lower bound and the upper bound is the largest transovarial transmission rate of Aedes mosquitoes across nodes.

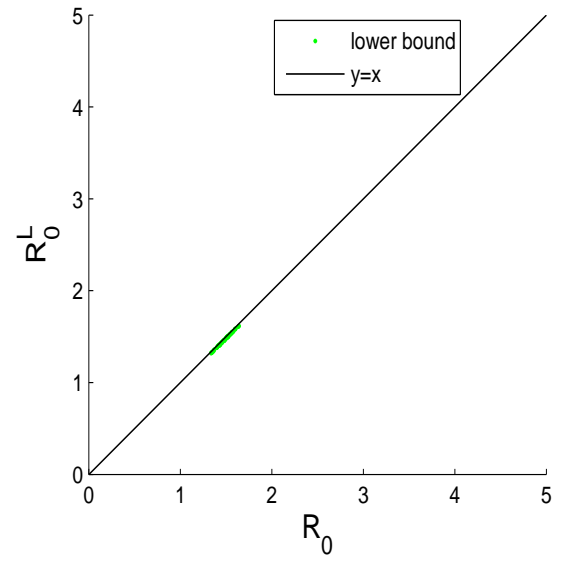

(a) The reproduction number and its lower bound with heterogeneous parameters.

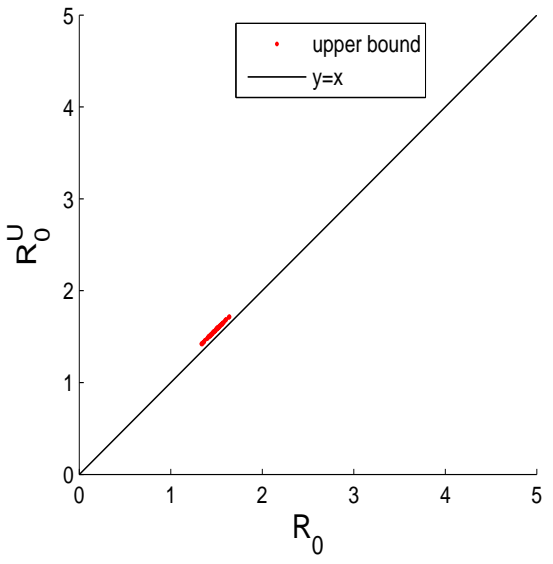

(b) The reproduction number and its upper bound with heterogeneous parameters.

Figure 1: The reproduction number and its lower and upper bounds computed using Theorem 1 for one hundred simulation runs in one hundred-node heterogeneous networks.

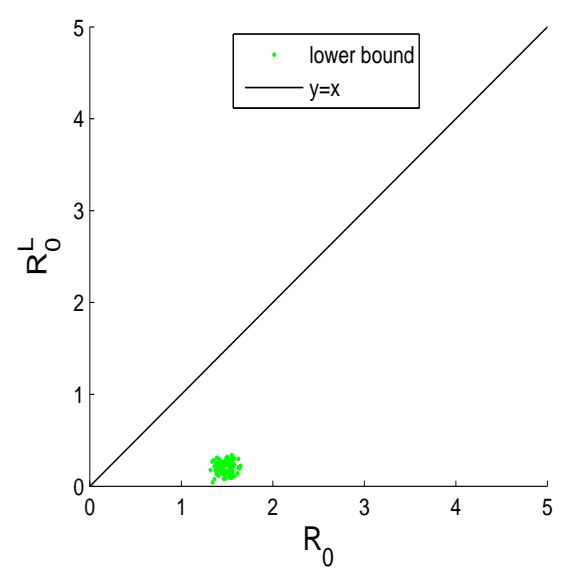

(a) The reproduction number and its lower bound with heterogeneous parameters.

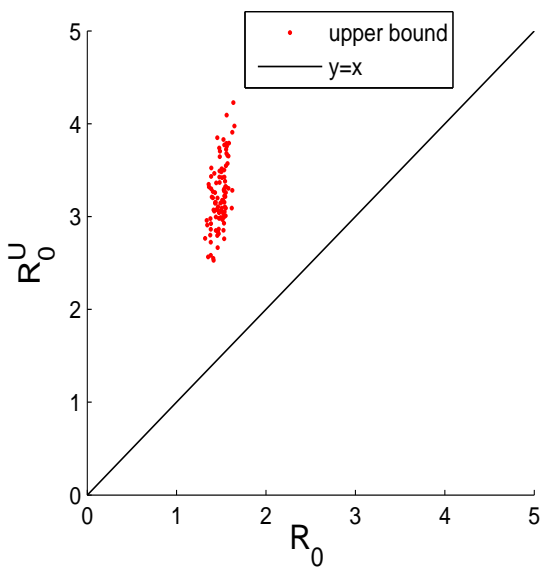

(b) The reproduction number and its upper bound with heterogeneous parameters.

Figure 2: The reproduction number and its lower and upper bounds computed using Theorem 2 for one hundred simulation runs in one hundred-node heterogeneous networks.

\subsection{Assessing the role of parameters on $R_{0}$}

As an example, a two-node network demonstrates how bounds of $R_{0}$ alter with livestock movement rates, if parameters $d_{2 i}, \gamma_{2 i}$, and $\mu_{2 i}$ are heterogeneous, i.e., at least one of inequalities $d_{2 i} \neq d_{2 j}$, $\gamma_{2 i} \neq \gamma_{2 j}, \mu_{2 i} \neq \mu_{2 j}$ holds for different $i$ and $j$. In this example, $M_{2}$ corresponds to the one in Equation 


\begin{tabular}{|c|c|c|c|}
\hline No. & parameter & $\begin{array}{l}\text { livestock movement } \\
\text { rates }\end{array}$ & $R_{0}$ \\
\hline 1 & $\beta_{12 i}>\beta_{12 j}, \beta_{21 i}>\beta_{21 j}, \beta_{23 i}>\beta_{23 j}, \quad \beta_{32 i}>\beta_{32 j}$ & $\omega_{2 j i}$ increases & increases \\
\hline & $y_{1}$ & $\omega_{2 i j}$ increases & decreases \\
\hline 2 & $d_{2 i}>d_{2 j}$ & $\omega_{j i}^{2}$ increases & decreases \\
\hline 3 & $\gamma_{2 i}>\gamma_{2 j}$ & $\omega_{2 j i}$ increases & decreases \\
\hline & & $\omega_{2 i j}$ increases & increases \\
\hline 4 & $\mu_{2 i}>\mu_{2 j}$ & $\begin{array}{l}\omega_{2 j i} \text { increases } \\
\omega_{2 i j} \text { increases }\end{array}$ & $\begin{array}{l}\text { decreases } \\
\text { increases }\end{array}$ \\
\hline
\end{tabular}

Table 1: Different scenarios for numerical simulations in four-node networks. Other parameters are kept the same and homogeneous across all nodes during all realizations. The superscripts $i, j=1,2,3,4$ and $i>j$.

(7) and $X_{2}=M_{2}+\oplus_{i=1}^{2}\left(\gamma_{2 i}+\mu_{2 i}-\varepsilon_{2 i}\right)$. Since $X_{2}, M_{2}$ are both diagonal dominant matrices, by Theorem $7, M_{2}^{-1}$ and $X_{2}^{-1}$ are both nonnegative matrices.

According to Proposition 4.3 in [37], $\rho\left(X_{2}^{-1} M_{2}^{-1}\right)$ is decreasing in $\omega_{212}$ if

$$
\omega_{212}\left(a_{2}-a_{1}\right)>\left(a_{1} c_{1}-a_{2} c_{2}\right)-\left(a_{2}-a_{1}\right) \omega_{221}
$$

and increasing otherwise, where $a_{1}=d_{21}+\varepsilon_{21}, a_{2}=d_{22}+\varepsilon_{22}, c_{1}=d_{21}+\gamma_{21}+\mu_{21}$ and $c_{2}=d_{22}+\gamma_{22}+\mu_{22}$. In the case that $a_{1}=a_{2}, \rho\left(X_{2}^{-1} M_{2}^{-1}\right)$ is decreasing in $\omega_{212}$ if $c_{2}>c_{1}$ and increasing otherwise. If $a_{1} \neq a_{2}, \omega_{212}^{*}:=\frac{a_{1} c_{1}-a_{2} c_{2}}{a_{2}-a_{1}}-\omega_{221}$ is a critical point of $\rho\left(X_{2}^{-1} M_{2}^{-1}\right)$. Moreover, $\rho\left(X_{2}^{-1} M_{2}^{-1}\right)$ reaches the maximum value at $\omega_{212}^{*}$ if $a_{2}>a_{1}$ and the minimum value at $\omega_{212}^{*}$ otherwise.

To evaluate the impact of networks with corresponding homogeneous parameters across all nodes on the value of $R_{0}$ computed using Equation (26), we construct three networks with three, four, and one hundred nodes, respectively. Simulation runs with varying livestock movement rates, and parameters in (33) and (37) held constant and homogeneous across nodes showed $R_{0}$ is not affected by livestock movement rates during one hundred runs per network. Moreover, the values and bounds of $R_{0}$ obtained through numerical simulations are the same for networks with three, four, and one hundred nodes. Through extensive numerical simulations, we have observed that $R_{0}$ does not depend on livestock movement rates or the number of nodes in a network when (33) and (37) hold.

We run scenarios (see Table 1) one hundred times for each four-node network to study the impact of livestock movement rates on $R_{0}$. During one hundred realizations for each scenario, we increase livestock movement rates while keeping remaining parameters constant and homogeneous across all nodes. In Scenario 1, we set contact rates $\beta_{12}, \beta_{21}, \beta_{23}$, and $\beta_{32}$ for node $i$ larger than respective parameters for node $j(i>j, i, j=1,2,3,4)$. During each run, $R_{0}$ increases while increasing livestock movement rates from node $j$ to node $i, \omega_{2 j i}$, and decreases while increasing livestock movement rates from node $i$ to node $j, \omega_{2 i j}$ (see Figure 3(a) and 3(b), respectively). In Scenario 2, under setting $d_{2 i}>d_{2 j}, R_{0}$ decreases when $\omega_{2 j i}$ increases, and increases when $\omega_{2 i j}$ increases (see Figure $4(\mathrm{a})$ and 4 (b), respectively). With livestock recovery rates $\gamma_{2 i}>\gamma_{2 j}$ in Scenario $3, R_{0}$ decreases when $\omega_{2 j i}$ increases, and increases when $\omega_{2 i j}$ increases (see Figure 5(a) and 5(b), respectively). Similarly, when livestock mortality rates $\mu_{2 i}>\mu_{2 j}$ in Scenario $4, R_{0}$ decreases when $\omega_{2 j i}$ increases, and increases with larger $\omega_{2 i j}$ (see Figure 6(a) and 6(b), respectively). Tuning the parameters in above scenarios yields $R_{0}$ from below 1 to above 1 . As a consequence, livestock movement rates are important in either leading to an epidemic outbreak or epidemic burnout.

\section{Results and discussions}

We propose an explicit expression of $R_{0}$, which is formulated as a function of vertical and horizontal transmission parameters shown in Equation (26). This formula facilitates computing $R_{0}$ for many 


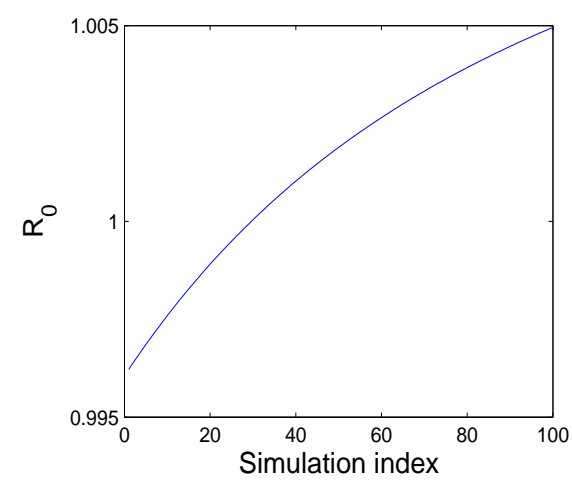

(a) As the livestock movement rate from node $j$ to node $i\left(\omega_{2 j i}\right)$ increases when $\beta_{12 i}>\beta_{12 j}, \beta_{21 i}>\beta_{21 j}, \beta_{23 i}>\beta_{23 j}$, and $\beta_{23 i}>\beta_{23 j}, R_{0}$ increases.

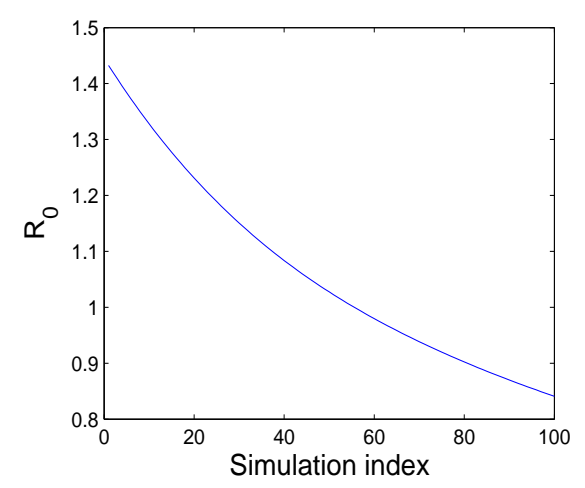

(b) As the livestock movement rate from node $i$ to node $j\left(\omega_{2 i j}\right)$ increases when $\beta_{12 i}>\beta_{12 j}, \beta_{21 i}>\beta_{21 j}, \beta_{23 i}>\beta_{23 j}$, and $\beta_{23 i}>\beta_{23 j}, R_{0}$ decreases.

Figure 3: The reproduction number for four-node networks with different contact rates during one hundred runs.

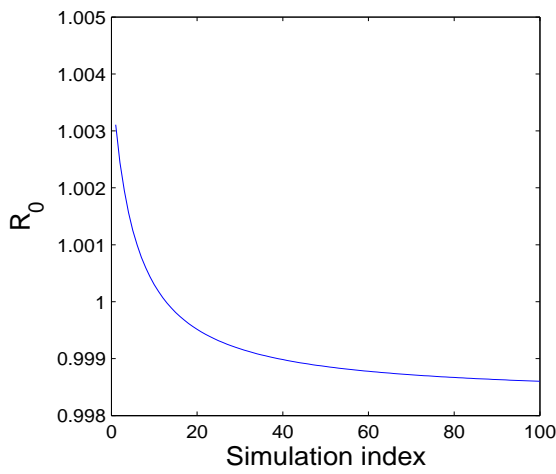

(a) As the livestock movement rate from node $j$ to node $i\left(\omega_{2 j i}\right)$ increases when $d_{2 i}>$ $d_{2 j}, R_{0}$ decreases.

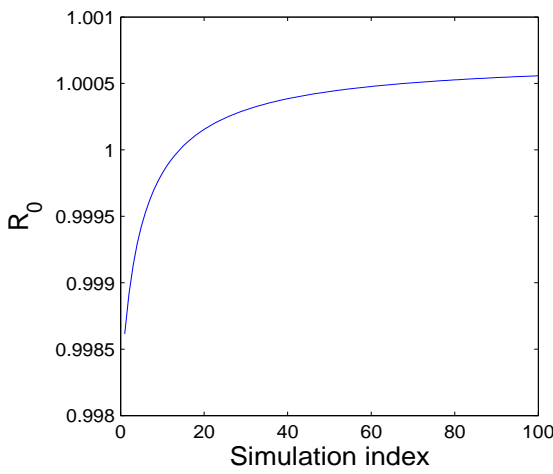

(b) As the livestock movement rate from node $i$ to node $j\left(\omega_{2 i j}\right)$ increases when $d_{2 i}>$ $d_{2 j}, R_{0}$ increases.

Figure 4: The reproduction number for four-node networks with different livestock death rates during one hundred runs.

diseases that involve both vertical and horizontal transmission by replacing the spectral radius of the original next generation matrix with that of a smaller size matrix. The lower bound of $R_{0}$ equals the reproduction number for horizontal transmission. We applied Equation (26) to the RVF model, deriving $R_{0}$ and its lower and upper bounds. We compared the tightness of different bounds, and analyzed the role of livestock movement rates and disease parameters on $R_{0}$ through numerical simulations.

The reproduction number for RVF meta-population model relates to the reproduction number for horizontal transmission, involving Aedes-livestock interaction and Culex-livestock interaction, and vertical transmission parameters. Different bounds of $R_{0}$ for heterogeneous networks are given by Theorem 1, Theorem 2, and Theorem 3 with decreasing tightness and increasing easiness. For homogeneous networks, the reproduction number for horizontal transmission in Equation (39) and bounds of $R_{0}$ given by Corollary 2 are proved independent of livestock movement rates, and equal to corresponding terms for homogeneous populations presented in [26]. The lower bound is the reproduction number for horizontal transmission and upper bound is the sum of the reproduction number for horizontal transmission and the largest transovarial transmission rate of Aedes mosquitoes among nodes.

Typically networks in the real world are heterogeneous. Rates of livestock death, incubation, mortality, recovery, and contact with mosquitoes can vary in different nodes due to climate, public health 


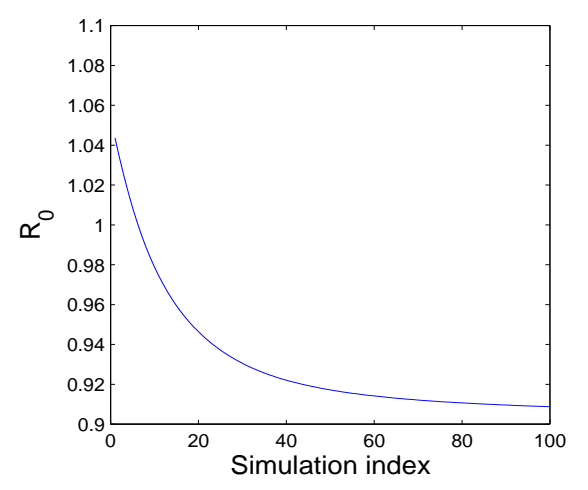

(a) As the livestock movement rate from node $j$ to node $i\left(\omega_{2 j i}\right)$ increases when $\gamma_{2 i}>$ $\gamma_{2 j}, R_{0}$ decreases.

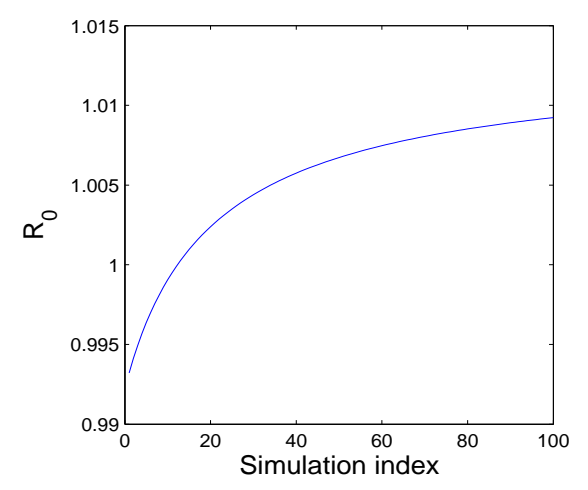

(b) As the livestock movement rate from node $i$ to node $j\left(\omega_{2 i j}\right)$ increases when $\gamma_{2 i}>$ $\gamma_{2 j}, R_{0}$ increases.

Figure 5: The reproduction number for four-node networks with different livestock recovery rates during one hundred runs.

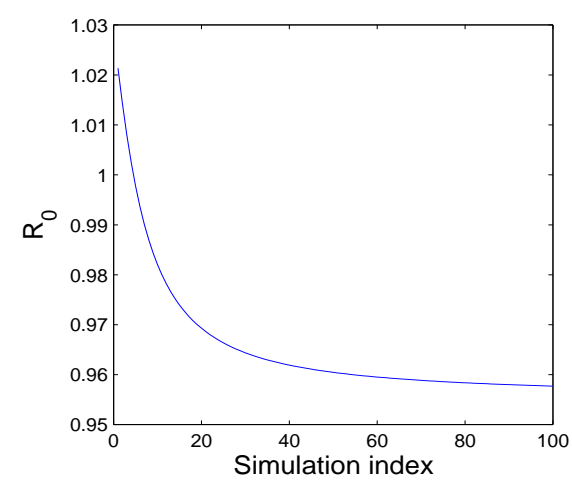

(a) As the livestock movement rate from node $j$ to node $i\left(\omega_{2 j i}\right)$ increases when $\mu_{2 i}>$ $\mu_{2 j}, R_{0}$ decreases.

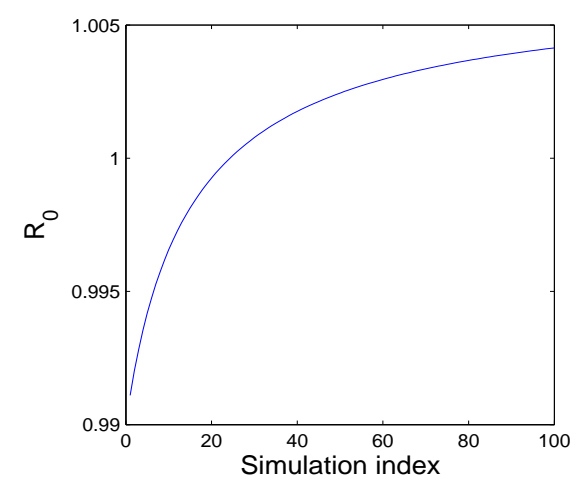

(b) As the livestock movement rate from node $i$ to node $j\left(\omega_{2 i j}\right)$ increases when $\mu_{2 i}>$ $\mu_{2 j}, R_{0}$ increases.

Figure 6: The reproduction number for four-node networks with different livestock mortality rates during one hundred runs.

facilities, environment, and/or type of nodes (e.g., death rates of livestock in feedlots are higher than those in livestock premises). Variations in weather may affect values of some mosquito parameters, e.g., rainfall affects mosquito birth rates, and temperature affects mosquito incubation rates. Even if weather conditions are homogeneous across all nodes, different genera and/or species of mosquitoes can exhibit different rates of incubation, contact, death, birth, and/or birth. Numerical simulations show livestock movement rates between different nodes only affect $R_{0}$ when the network is spatially heterogeneous regarding parameters. Changing livestock movement rates on heterogeneous networks results in $R_{0}$ varying between values below and above the critical value 1 . When other parameters remain homogeneous and constant, increasing livestock movement rates from nodes with smaller contact rates to those with larger contact rates increases $R_{0}$. If livestock movement rates are increased from nodes with smaller livestock death rates (or recovery rates, or mortality rates) to nodes with larger livestock death rates (or recovery rates, or mortality rates), $R_{0}$ decreases. This observation helps us better envision effective mitigation strategies executing movement bans between some nodes and in some directions.

Whatever heterogeneity exists between nodes, our same mathematical model in Equations (18) 
through (23), and the explicit expression of $R_{0}$ in (26), are applicable. Our formula for $R_{0}$ presented in this paper can be used for numerous diseases models aside from RVF.

Our work on RVF contributes computing $R_{0}$ accurately by taking into account vertical transmission, which is important but ignored by modelers. We simplified the derivation of $R_{0}$ by computing the spectral radius of a smaller size matrix than the original next generation matrix. Bounds of $R_{0}$ facilitate estimating $R_{0}$ of RVF metapopulation model. The simulation results on livestock movement rates and parameters are helpful in developing efficient mitigation strategies for RVF.

\section{Acknowledgments}

This work has been supported by Kansas Bioscience Authority (KBA) and the DHS Center of Excellence for Emerging and Zoonotic Animal Diseases (CEEZAD). We are grateful to the effort made by the anonymous reviewers. We would like to give thanks to Alice Trussell and Andrea Engelken for help on English proofreading.

\section{References}

[1] M. Salmani, P. van den Driessche, A model for disease transmission in a patchy environment, Discrete and Continuous Dynamical Systems-Series B 6 (2006) 185-202.

[2] W. Wang, X. Q. Zhao, An epidemic model in a patchy environment, Mathematical biosciences 190 (2004) 97-112.

[3] J. Arino, J. R. Davis, D. Hartley, R. Jordan, J. M. Miller, P. van den Driessche, A multi-species epidemic model with spatial dynamics, Mathematical Medicine and Biology 22 (2005) 129-142.

[4] R. B. Stothers, Climatic and demographic consequences of the massive volcanic eruption of 1258, Climatic Change 45 (2000) 361-374.

[5] L. R. Petersen, J. T. Roehrig, West Nile virus: A reemerging global pathogen, Emerging Infectious Diseases 7 (2001) 611-614.

[6] World Health Organization Multicentre Collaborative Network for Severe and Acute Respiratory Syndrome Diagnosis, A multicentre collaboration to investigate the cause of severe acute respiratory syndrome, Lancet 361 (2003) 1730-1733.

[7] J. Arino, R. Jordan, P. van den Driessche, Quarantine in a multi-species epidemic model with spatial dynamics, Mathematical biosciences 206 (2007) 46-60.

[8] S. Busenberg, K. L. Cooke, Models of vertically transmitted diseases with sequential- continuous dynamics, Academic press, 1982.

[9] M. El-Doma, Analysis of an age-dependent SIS epidemic model with vertical transmission and proportionate mixing assumption, Mathematical and Computer Modelling 29 (1999) 31-43.

[10] M. Y. Li, H. L. Smith, L. C. Wang, Global dynamics of an SEIR epidemic model with vertical transmission, SIAM Journal on Applied Mathematics 62 (2001) 58-69.

[11] S. Busenberg, K. L. Cooke, Vertically transmitted diseases: models and dynamics, Berlin New York: Springer, 1993.

[12] N. Chitnis, J. M. Hyman, C. A. Manore, Modelling vertical transmission in vector-borne diseases with applications to Rift Valley fever, Journal of biological dynamics 7 (2013) 11-40. 
[13] R. M. Anderson, R. M. May, The population-dynamics of micro-parasites and their invertebrate hosts, Philosophical Transactions of the Royal Society of London Series B-Biological Sciences 291 (1981) 451-524.

[14] S. Busenberg, K. L. Cooke, The population-dynamics of two vertically transmitted infections, Theoretical population biology 33 (1988) 181-198.

[15] P. Rohani, D. J. Earn, B. T. Grenfell, Opposite patterns of synchrony in sympatric disease metapopulations, Science 286 (1999) 968-971.

[16] N. J. Gotelli, C. M. Taylor, Testing metapopulation models with stream-fish assemblages, Evolutionary Ecology Research 1 (1999) 835-845.

[17] C. M. Taylor, R. J. Hall, Metapopulation models for seasonally migratory animals, Biology letters 8 (2012) 477-480.

[18] J. Arino, P. van den Driessche, The basic reproduction number in a multi-city compartmental epidemic model, Positive Systems, Proceedings 294 (2003) 135-142.

[19] M. J. Keeling, M. E. J. Woolhouse, D. J. Shaw, L. Matthews, M. Chase-Topping, D. T. Haydon, S. J. Cornell, J. Kappey, J. Wilesmith, B. T. Grenfell, Dynamics of the 2001 UK foot and mouth epidemic: Stochastic dispersal in a heterogeneous landscape, Science 294 (2001) 813-817.

[20] O. Diekmann, J. A. P. Heesterbeek, M. G. Roberts, The construction of next-generation matrices for compartmental epidemic models, Journal of the Royal Society Interface 7 (2010) 873-885.

[21] O. Diekmann, J. Heesterbeek, J. Metz, On the definition and the computation of the basic reproduction ratio $\mathrm{R} 0$ in models for infectious diseases in heterogeneous populations, Journal of Mathematical Biology 28 (1990) 365-382.

[22] O. Diekmann, J. A. P. Heesterbeek, Mathematical Epidemiology of Infectious Diseases, Wiley Chichester, 2000.

[23] P. van den Driessche, J. Watmough, Reproduction numbers and sub-threshold endemic equilibria for compartmental models of disease transmission, Mathematical biosciences 180 (2002) 29-48.

[24] J. Li, D. Blakeley, R. Smith?, The failure of R0, Computational and Mathematical Methods in Medicine 2011 (2011).

[25] M. Lipsitch, M. A. Nowak, D. Ebert, R. M. May, The population dynamics of vertically and horizontally transmitted parasites, Proceedings.Biological sciences / The Royal Society 260 (1995) $321-327$.

[26] L. Xue, H. M. Scott, L. W. Cohnstaedt, C. Scoglio, A network-based meta-population approach to model Rift Valley fever epidemics, Journal of Theoretical Biology 306 (2012) 129-144.

[27] J. Heffernan, R. Smith, L. Wahl, Perspectives on the basic reproductive ratio, Journal of the Royal Society Interface 2 (2005) 281-293.

[28] R. J. Plemmons, M-matrix characterizations 1: Nonsingular M-matrices, Linear Algebra and its Applications 18 (1977) 175-188.

[29] R. Flick, M. Bouloy, et al., Rift Valley fever virus, Current molecular medicine 5 (2005) 827-834.

[30] V. Chevalier, M. Pépin, L. Plée, R. Lancelot, Rift Valley fever-a threat for Europe?, Euro Surveill. 15 (2010) 19506. 
[31] M. B. Crabtree, R. J. K. Crockett, B. H. Bird, S. T. Nichol, B. R. Erickson, B. J. Biggerstaff, K. Horiuchi, B. R. Miller, Infection and transmission of Rift Valley fever viruses lacking the NSs and/or NSm genes in mosquitoes: Potential role for NSm in mosquito infection, Plos Neglected Tropical Diseases 6 (2012) e1639.

[32] G. Gerdes, Rift Valley fever, Veterinary Clinics of North America-Food Animal Practice 18 (2002) $549-555$.

[33] R. F. Breiman, B. Minjauw, S. K. Sharif, P. Ithondeka, M. K. Njenga, Rift Valley fever: scientific pathways toward public health prevention and response, The American Journal of Tropical Medicine and Hygiene 83 (2010) 1-4.

[34] K. J. Linthicum, A. Anyamba, C. J. Tucker, P. W. Kelley, M. F. Myers, C. J. Peters, Climate and satellite indicators to forecast Rift Valley fever epidemics in Kenya, Science 285 (1999) 397-400.

[35] J. Arino, Diseases in metapopulations, Modeling and dynamics of infectious diseases 11 (2009) 64-122.

[36] D. Gao, S. Ruan, An SIS patch model with variable transmission coefficients, Mathematical biosciences 232 (2011) 110-115.

[37] D. Gao, S. Ruan, A multipatch malaria model with logistic growth populations, SIAM Journal on Applied Mathematics 72 (2012) 819-841.

[38] Y. H. Hsieh, P. van den Driessche, L. Wang, Impact of travel between patches for spatial spread of disease, Bulletin of mathematical biology 69 (2007) 1355-1375.

[39] F. R. Adler, The effects of averaging on the basic reproduction ratio, Mathematical biosciences 111 (1992) 89-98.

[40] J. Cohen, Random evolutions and the spectral radius of a non-negative matrix, Math. Proc. Camb. Phil. Soc. 86 (1979) 345-350.

[41] G. Cheng, X. Cheng, T. Huang, T. Tam, Some bounds for the spectral radius of the Hadamard product of matrices, Applied Mathematics E-Notes 5 (2005) 202-209.

[42] D. V. Canyon, J. L. K. Hii, R. Muller, The frequency of host biting and its effect on oviposition and survival in Aedes aegypti (Diptera: Culicidae), Bulletin of entomological research 89 (1999) $35-39$.

[43] R. O. Hayes, C. H. Tempelis, A. D. Hess, W. C. Reeves, Mosquito host preference studies in Hale County, Texas, American Journal of Tropical Medicine and Hygiene 22 (1973) 270-277.

[44] C. J. Jones, J. E. Lloyd, Mosquitos feeding on sheep in southeastern Wyoming, Journal of the American Mosquito Control Association 1 (1985) 530-532.

[45] L. A. Magnarelli, Host feeding patterns of Connecticut mosquitos (Diptera: Culicidae), American Journal of Tropical Medicine and Hygiene 26 (1977) 547-552.

[46] H. D. Pratt, C. G. Moore, Vector-borne disease control: mosquitoes of public health importance and their control, U.S. Department of Health and Human Services, Atlanta, GA, 1993.

[47] M. J. Turell, C. L. Bailey, J. R. Beaman, Vector competence of a Houston, Texas strain of Aedes Albopictus for Rift Valley fever virus, Journal of the American Mosquito Control Association 4 (1988) 94-96. 
[48] M. J. Turell, M. E. Faran, M. Cornet, C. L. Bailey, Vector competence of senegalese Aedes fowleri (Diptera: Culicidae) for Rift Valley fever virus, Journal of Medical Entomology 25 (1988) 262-266.

[49] M. J. Turell, C. L. Bailey, Transmission studies in mosquitoes (Diptera: Culicidae) with disseminated Rift Valley fever virus infections, Journal of Medical Entomology 24 (1987) 11-18.

[50] J. W. Wekesa, B. Yuval, R. K. Washino, Multiple blood feeding by Anopheles freeborni and Culex tarsalis (Diptera: Culicidae): Spatial and temporal variation, Journal of Medical Entomology 34 (1997) 219-225.

[51] B. J. Erasmus, J. A. W. Coetzer, The symptomatology and pathology of Rift Valley fever in domestic animals, Contrib. Epidemiol. Biostat. 3 (1981) 77-82.

[52] S. C. Mpeshe, H. Haario, J. M. Tchuenche, A mathematical model of Rift Valley fever with human host, Acta Biotheoretica 59 (2011) 231-250.

[53] M. Bates, The natural history of mosquitoes, American Journal of Public Health 39 (1949) 1592.

[54] C. G. Moore, R. G. McLean, C. J. Mitchell, R. S. Nasci, T. F. Tsai, C. H. Caslisher, A. A. Marfin, P. S. Moorse, D. J. Gubler, Guidelines for Arbovirus Surveillance Programs in the United Sates, Centers for Disease Control and Prevention, April 1993.

[55] O. M. Radostits, Herd Healthy: Food Animal Production Medicine, Saunders, third edition, 2001.

[56] C. J. Peters, K. J. Linthicum, Rift Valley fever, in: Handbook of Zoonoses. Second Edition. Section B: Viral. G.B. Beran (Ed.), CRC Press, Inc., Boca Raton, Fl, 1994, pp. 125-138.

[57] J. E. Freier, L. Rosen, Vertical transmission of dengue viruses by mosquitoes of the aedes scutellaris group, The American Journal of Tropical Medicine and Hygiene 37 (1987) 640-647.

\section{Appendix}

Proof of Theorem 1. The left inequality is the same as (17). We now show that the right inequality holds. By (28) and (25),

$$
\begin{gathered}
-W\left(\oplus_{i=1}^{n} \theta_{1 i}^{-1}\right) U V_{H}^{-1}=\left[\begin{array}{cc}
0_{4 n \times 4 n} & 0_{4 n \times 4 n} \\
Y & Z
\end{array}\right], \text { where } \\
Y=\left[\begin{array}{cc}
X_{1}^{-1}\left(\oplus_{i=1}^{n}\left(b_{1 i} q_{1 i} \varepsilon_{1 i}\right)\right) M_{1}^{-1} & 0_{n \times 3 n} \\
0_{3 n \times n} & 0_{3 n \times 3 n}
\end{array}\right], \quad Z=\left[\begin{array}{cc}
\left(\oplus_{i=1}^{n}\left(b_{1 i} q_{1 i}\right)\right) X_{1}^{-1} & 0_{n \times 3 n} \\
0_{3 n \times n} & 0_{3 n \times 3 n}
\end{array}\right] .
\end{gathered}
$$

Note that $X_{1}$ and $X_{1}^{-1}$ are diagonal matrices. Moreover, the nonzero eigenvalues of $-W\left(\oplus_{i=1}^{n} \theta_{1 i}^{-1}\right) U V_{H}^{-1}$ are diagonal entries of $\left(\oplus_{i=1}^{n}\left(b_{1 i} q_{1 i}\right)\right) X_{1}^{-1}$. Hence, $-W\left(\oplus_{i=1}^{n} \theta_{1 i}^{-1}\right) U V_{H}^{-1}=\mathcal{P} \mathcal{D} \mathcal{P}^{-1}$ for some $\mathcal{P}$. Here

$$
\mathcal{D}=\left[\begin{array}{cc}
0_{4 n \times 4 n} & 0_{4 n \times 4 n} \\
0_{4 n \times 4 n} & \mathcal{Q}
\end{array}\right], \quad \mathcal{Q}=\left[\begin{array}{cc}
0_{3 n \times 3 n} & 0_{3 n \times n} \\
0_{n \times 3 n} & \left(\oplus_{i=1}^{n}\left(b_{1 i} q_{1 i}\right)\right) X_{1}^{-1}
\end{array}\right] .
$$

From linear algebra, each column of $\mathcal{P}$ can be chosen as an eigenvector of $-W\left(\oplus_{i=1}^{n} \theta_{1 i}^{-1}\right) U V_{H}^{-1}$. By direct calculation,

$$
\mathcal{P}=\left[\begin{array}{cc}
\mathcal{H}_{4 n \times 4 n} & 0 \\
\mathcal{J}_{4 n \times 4 n} & \mathcal{L}_{4 n \times 4 n}
\end{array}\right], \quad \text { where } \quad \mathcal{H}=\left[\begin{array}{cc}
\left(\oplus_{i=1}^{n}\left(b_{1 i} q_{1 i}\right)\right) X_{1}^{-1} & 0_{n \times 3 n} \\
0_{3 n \times n} & I_{3 n \times 3 n}
\end{array}\right],
$$




$$
\mathcal{L}=\left[\begin{array}{cc}
0_{n \times 3 n} & I_{n \times n} \\
I_{3 n \times 3 n} & 0_{3 n \times n}
\end{array}\right], \quad \mathcal{J}=\left[\begin{array}{cc}
-\left(\oplus_{i=1}^{n}\left(b_{1 i} q_{1 i} \varepsilon_{1 i}\right)\right) X_{1}^{-1} M_{1}^{-1} & 0_{n \times 3 n} \\
0_{3 n \times n} & 0_{3 n \times 3 n}
\end{array}\right] .
$$

Since $F_{H} V_{H}^{-1}-W\left(\oplus_{i=1}^{n} \theta_{1 i}^{-1}\right) U V_{H}^{-1}=\mathcal{P}\left(\mathcal{P}^{-1} F_{H} V_{H}^{-1} \mathcal{P}+\mathcal{D}\right) \mathcal{P}^{-1}$, we have

$$
\rho\left(F V^{-1}\right)=\rho\left(F_{H} V_{H}^{-1}-W\left(\oplus_{i=1}^{n} \theta_{1 i}^{-1}\right) U V_{H}^{-1}\right)=\rho\left(\mathcal{P}^{-1} F_{H} V_{H}^{-1} \mathcal{P}+\mathcal{D}\right) .
$$

We clam that $\mathcal{P}^{-1} F_{H} V_{H}^{-1} \mathcal{P}$ is a nonnegative matrix. By calculation,

$$
\mathcal{P}^{-1}=\left[\begin{array}{cc}
\mathcal{H}^{-1} & 0 \\
-\mathcal{L}^{-1} \mathcal{J H}^{-1} & \mathcal{L}^{-1}
\end{array}\right], \mathcal{H}^{-1}=\left[\begin{array}{cc}
\left(\oplus_{i=1 \frac{1}{b_{1 i} q_{1 i}}}^{n}\right) X_{1} & 0_{n \times 3 n} \\
0_{3 n \times n} & I_{3 n \times 3 n}
\end{array}\right], \quad \mathcal{L}^{-1}=\left[\begin{array}{cc}
0_{3 n \times n} & I_{3 n \times 3 n} \\
I_{n \times n} & 0_{n \times 3 n}
\end{array}\right] .
$$

It is clear that $\mathcal{H}^{-1}, \mathcal{L}^{-1}$, and $-\mathcal{L}^{-1} \mathcal{J} \mathcal{H}^{-1}$ are all nonnegative matrices. Hence, $\mathcal{P}^{-1}$ is a nonnegative matrix. We now show that $F_{H} V_{H}^{-1} \mathcal{P}$ is a nonnegative matrix.

$$
F_{H} V_{H}^{-1} \mathcal{P}=\left[\begin{array}{cc}
\mathcal{A}\left(\oplus_{k=1}^{4} \mathcal{Z}_{k}\right) \mathcal{H}+\mathcal{A}\left(\oplus_{k=1}^{4} X_{k}^{-1}\right) \mathcal{J} & \mathcal{A}\left(\oplus_{k=1}^{4} X_{k}^{-1}\right) \mathcal{L} \\
0
\end{array}\right]
$$

where $\mathcal{A}\left(\oplus_{k=1}^{4} X_{k}^{-1}\right) \mathcal{L}$ is a nonnegative matrix and $\mathcal{Z}_{k}=X_{k}^{-1}\left(\oplus_{i=1}^{n} \varepsilon_{k i}\right) M_{k}^{-1}$. Furthermore, the only possible negative entries of $\mathcal{A}\left(\oplus_{k=1}^{4} \mathcal{Z}_{k}\right) \mathcal{H}+\mathcal{A}\left(\oplus_{k=1}^{4} X_{k}^{-1}\right) \mathcal{J}$ are in its $(2,1)$ and $(4,1)$ blocks. But the block in $(2,1)$-entry is

$$
\mathcal{A}_{21} X_{1}^{-1}\left(\oplus_{i=1}^{n} \varepsilon_{1 i}\right) M_{1}^{-1}\left(\oplus_{i=1}^{n}\left(b_{1 i} q_{1 i}\right)\right) X_{1}^{-1}+\mathcal{A}_{21} X_{1}^{-1}\left(-\oplus_{i=1}^{n}\left(b_{1 i} q_{1 i} \varepsilon_{1 i}\right)\right) X_{1}^{-1} M_{1}^{-1}=0 .
$$

By assumption, $X_{1}$ and $M_{1}$ are both diagonal matrices. The last equality follows $X_{1}^{-1} M_{1}^{-1}=M_{1}^{-1} X_{1}^{-1}$. Similarly, the block in $(4,1)$-entry is

$$
\mathcal{A}_{41} X_{1}^{-1}\left(\oplus_{i=1}^{n} \varepsilon_{1 i}\right) M_{1}^{-1}\left(\oplus_{i=1}^{n}\left(b_{1 i} q_{1 i}\right)\right) X_{1}^{-1}+\mathcal{A}_{41} X_{1}^{-1}\left(-\oplus_{i=1}^{n}\left(b_{1 i} q_{1 i} \varepsilon_{1 i}\right)\right) X_{1}^{-1} M_{1}^{-1}=0 .
$$

Hence, $F_{H} V_{H}^{-1} \mathcal{P}$ is a nonnegative matrix. This proves the claim. By Theorem 2 in [40], we have

$$
\rho\left(F V^{-1}\right) \leq \rho\left(\mathcal{P}^{-1} F_{H} V_{H}^{-1} \mathcal{P}\right)+\rho(\mathcal{D})=\rho\left(F_{H} V_{H}^{-1}\right)+\rho(\mathcal{D}) .
$$

Since $X_{1}=\oplus_{i=1}^{n} \frac{d_{1 i} N_{1 i}^{0}}{K_{1}}$ and $N_{1 i}^{0}=\frac{b_{1 i} K_{1}}{d_{1 i}}$, we further have

$$
\rho(\mathcal{D})=\rho\left(-W\left(\oplus_{i=1}^{n} \theta_{1 i}^{-1}\right) U V_{H}^{-1}\right)=\rho\left(\left(\oplus_{i=1}^{n}\left(b_{1 i} q_{1 i}\right)\right) X_{1}^{-1}\right)=\rho\left(\oplus_{i=1}^{n} q_{1 i}\right) \leqslant \max _{i}\left(q_{1 i}\right) .
$$

Therefore,

$$
\rho\left(F_{H} V_{H}^{-1}\right) \leq R_{0}=\rho\left(F V^{-1}\right) \leq \rho\left(F_{H} V_{H}^{-1}\right)+\max _{i}\left(q_{1 i}\right)
$$

This finishes the proof.

Proof of Theorem 2. By Equations (25) and (27),

$$
F_{H} V_{H}^{-1}=\left[\begin{array}{cc}
\mathcal{A}\left(\oplus_{k=1}^{4} \mathcal{Z}_{k}\right) & \mathcal{A}\left(\oplus_{k=1}^{4} X_{k}^{-1}\right) \\
0 & 0
\end{array}\right]
$$

Then

$$
R_{0}^{H}=\rho\left(F_{H} V_{H}^{-1}\right)=\rho\left(\mathcal{A}\left(\oplus_{k=1}^{4} \mathcal{Z}_{k}\right)\right) .
$$

By Equation (29),

$$
\mathcal{A}\left(\oplus_{k=1}^{4} \mathcal{Z}_{k}\right)=\left[\begin{array}{cccc}
0 & \mathcal{A}_{12} \mathcal{Z}_{2} & 0 & 0 \\
\mathcal{A}_{21} \mathcal{Z}_{1} & 0 & \mathcal{A}_{23} \mathcal{Z}_{3} & 0 \\
0 & \mathcal{A}_{32} \mathcal{Z}_{2} & 0 & 0 \\
\mathcal{A}_{41} \mathcal{Z}_{1} & \mathcal{A}_{42} \mathcal{Z}_{2} & \mathcal{A}_{43} \mathcal{Z}_{3} & 0
\end{array}\right]=:\left[\begin{array}{cccc}
0 & \mathcal{B}_{1} & 0 & 0 \\
\mathcal{B}_{2} & 0 & \mathcal{B}_{3} & 0 \\
0 & \mathcal{B}_{4} & 0 & 0 \\
\mathcal{B}_{5} & \mathcal{B}_{6} & \mathcal{B}_{7} & 0
\end{array}\right]
$$


To compute the eigenvalues of $\mathcal{A}\left(\oplus_{k=1}^{4} \mathcal{Z}_{k}\right)$, we first calculate the characteristic polynomial of $\mathcal{A}\left(\oplus_{k=1}^{4} \mathcal{Z}_{k}\right)$ as follows.

$$
\begin{aligned}
& \left|\lambda I_{4 n}-\mathcal{A}\left(\oplus_{k=1}^{4} \mathcal{Z}_{k}\right)\right|=\left|\begin{array}{cccc}
\lambda I_{n} & -\mathcal{B}_{1} & 0 & 0 \\
-\mathcal{B}_{2} & \lambda I_{n} & -\mathcal{B}_{3} & 0 \\
0 & -\mathcal{B}_{4} & \lambda I_{n} & 0 \\
-\mathcal{B}_{5} & -\mathcal{B}_{6} & -\mathcal{B}_{7} & \lambda I_{n}
\end{array}\right|=\lambda^{n}\left|\begin{array}{ccc}
\lambda I_{n} & -\mathcal{B}_{1} & 0 \\
-\mathcal{B}_{2} & \lambda I_{n} & -\mathcal{B}_{3} \\
0 & -\mathcal{B}_{4} & \lambda I_{n}
\end{array}\right| \\
& =\lambda^{n}\left|\left[\begin{array}{ccc}
I_{n} & \lambda \mathcal{B}_{2}^{-1} & 0 \\
0 & I_{n} & 0 \\
0 & 0 & I_{n}
\end{array}\right]\left[\begin{array}{ccc}
\lambda I_{n} & -\mathcal{B}_{1} & 0 \\
-\mathcal{B}_{2} & \lambda I_{n} & -\mathcal{B}_{3} \\
0 & -\mathcal{B}_{4} & \lambda I_{n}
\end{array}\right]\right|=\lambda^{n}\left|\left[\begin{array}{ccc}
0 & -\mathcal{B}_{1}+\lambda^{2} \mathcal{B}_{2}^{-1} & -\lambda \mathcal{B}_{2}^{-1} \mathcal{B}_{3} \\
-\mathcal{B}_{2} & \lambda I_{n} & -\mathcal{B}_{3} \\
0 & -\mathcal{B}_{4} & \lambda I_{n}
\end{array}\right]\right| \\
& =\lambda^{n}\left|\mathcal{B}_{2}\right|\left|\begin{array}{cc}
-\mathcal{B}_{1}+\lambda^{2} \mathcal{B}_{2}^{-1} & -\lambda \mathcal{B}_{2}^{-1} \mathcal{B}_{3} \\
-\mathcal{B}_{4} & \lambda I_{n}
\end{array}\right|=\lambda^{n}\left|\mathcal{B}_{2}\right|\left|\left[\begin{array}{cc}
-\mathcal{B}_{1}+\lambda^{2} \mathcal{B}_{2}^{-1} & -\lambda \mathcal{B}_{2}^{-1} \mathcal{B}_{3} \\
-\mathcal{B}_{4} & \lambda I_{n}
\end{array}\right]\left[\begin{array}{cc}
I_{n} & \lambda \mathcal{B}_{4}^{-1} \\
0 & I_{n}
\end{array}\right]\right| \\
& =\lambda^{n}\left|\mathcal{B}_{2}\right|\left|\begin{array}{cc}
-\mathcal{B}_{1}+\lambda^{2} \mathcal{B}_{2}^{-1} & -\lambda\left(\mathcal{B}_{1} \mathcal{B}_{4}^{-1}-\lambda^{2} \mathcal{B}_{2}^{-1} \mathcal{B}_{4}^{-1}+\mathcal{B}_{2}^{-1} \mathcal{B}_{3}\right) \\
-\mathcal{B}_{4} & 0
\end{array}\right| \\
& =\lambda^{n}\left|\mathcal{B}_{2}\right|\left|\begin{array}{cc}
-\lambda\left(\mathcal{B}_{1} \mathcal{B}_{4}^{-1}-\lambda^{2} \mathcal{B}_{2}^{-1} \mathcal{B}_{4}+\mathcal{B}_{2}^{-1} \mathcal{B}_{3}\right) & \mathcal{B}_{1}-\lambda^{2} \mathcal{B}_{2}^{-1} \\
0 & \mathcal{B}_{4}
\end{array}\right| \\
& =\lambda^{n}\left|\mathcal{B}_{2}\right|\left|\mathcal{B}_{4}\right|\left|-\lambda\left(\mathcal{B}_{1} \mathcal{B}_{4}^{-1}-\lambda^{2} \mathcal{B}_{2}^{-1} \mathcal{B}_{4}^{-1}+\mathcal{B}_{2}^{-1} \mathcal{B}_{3}\right)\right| \\
& =\lambda^{2 n}\left|\mathcal{B}_{2}\right|\left|\mathcal{B}_{4}\right|\left|\lambda^{2} \mathcal{B}_{2}^{-1} \mathcal{B}_{4}^{-1}-\left(\mathcal{B}_{1} \mathcal{B}_{4}^{-1}+\mathcal{B}_{2}^{-1} \mathcal{B}_{3}\right)\right| \\
& =\lambda^{2 n}\left|\mathcal{B}_{2}\right|\left|\mathcal{B}_{4}\right|\left|\mathcal{B}_{2}^{-1}\right|\left|\mathcal{B}_{4}^{-1}\right|\left|\lambda^{2} I_{n}-\left(\mathcal{B}_{4} \mathcal{B}_{2} \mathcal{B}_{1} \mathcal{B}_{4}^{-1}+\mathcal{B}_{4} \mathcal{B}_{3}\right)\right| \\
& =\lambda^{2 n}\left|\lambda^{2} I_{n}-\left(\mathcal{B}_{4} \mathcal{B}_{2} \mathcal{B}_{1} \mathcal{B}_{4}^{-1}+\mathcal{B}_{4} \mathcal{B}_{3}\right)\right| \text {. }
\end{aligned}
$$

Matrix $\mathcal{A}\left(\oplus_{k=1}^{4} \mathcal{Z}_{k}\right)$ has $2 n$ zero eigenvalues. The spectral radius of $\mathcal{A}\left(\oplus_{k=1}^{4} \mathcal{Z}_{k}\right)$ is the square root of the spectral radius of $\mathcal{B}_{4} \mathcal{B}_{2} \mathcal{B}_{1} \mathcal{B}_{4}^{-1}+\mathcal{B}_{4} \mathcal{B}_{3}$. By Equation (42), we obtain

$$
\left.\rho\left(F_{H} V_{H}^{-1}\right)=\sqrt{\rho\left(\mathcal{B}_{4} \mathcal{B}_{2} \mathcal{B}_{1} \mathcal{B}_{4}^{-1}+\mathcal{B}_{4} \mathcal{B}_{3}\right)}=\sqrt{\rho\left(\mathcal{B}_{4}\left(\mathcal{B}_{2} \mathcal{B}_{1}+\mathcal{B}_{3} \mathcal{B}_{4}\right) \mathcal{B}_{4}^{-1}\right.}\right)=\sqrt{\rho\left(\mathcal{B}_{2} \mathcal{B}_{1}+\mathcal{B}_{3} \mathcal{B}_{4}\right)} .
$$

Recall that $\mathcal{A}_{21}, \mathcal{A}_{12}, X_{1}, M_{1}, \mathcal{A}_{23}, \mathcal{A}_{32}, M_{3}, X_{3}$ are all diagonal matrices. By the assumption that $\varepsilon_{2 i}=\varepsilon_{2}$, for all $i$, we obtain

$$
\begin{aligned}
& \mathcal{B}_{2} \mathcal{B}_{1}=\left(\oplus_{i=1}^{n} \varepsilon_{1 i} \varepsilon_{2}\right) \mathcal{A}_{21} X_{1}^{-1} M_{1}^{-1} \mathcal{A}_{12} X_{2}^{-1} M_{2}^{-1}=\left(\oplus_{i=1}^{n} \frac{\varepsilon_{2} \varepsilon_{1 i} \beta_{12 i} \beta_{21 i}}{b_{1 i}\left(b_{1 i}+\varepsilon_{1 i}\right)}\right) X_{2}^{-1} M_{2}^{-1} \\
& \mathcal{B}_{3} \mathcal{B}_{4}=\left(\oplus_{i=1}^{n} \varepsilon_{2} \varepsilon_{3 i}\right) \mathcal{A}_{23} X_{3}^{-1} M_{3}^{-1} \mathcal{A}_{32} X_{2}^{-1} M_{2}^{-1}=\left(\oplus_{i=1}^{n} \frac{\varepsilon_{2} \varepsilon_{3 i} \beta_{32 i} \beta_{23 i}}{b_{3 i}\left(b_{3 i}+\varepsilon_{3 i}\right)}\right) X_{2}^{-1} M_{2}^{-1}
\end{aligned}
$$

By the definition of $\chi_{i}$ in (32), we have

$$
\min _{i}\left(\chi_{i}\right) \rho\left(X_{2}^{-1} M_{2}^{-1}\right) \leqslant \rho\left(\mathcal{B}_{2} \mathcal{B}_{1}+\mathcal{B}_{3} \mathcal{B}_{4}\right) \leqslant \max _{i}\left(\chi_{i}\right) \rho\left(X_{2}^{-1} M_{2}^{-1}\right) .
$$

Therefore,

$$
\sqrt{\min _{i}\left(\chi_{i}\right) \rho\left(X_{2}^{-1} M_{2}^{-1}\right)} \leqslant \rho\left(F_{H} V_{H}^{-1}\right) \leqslant \sqrt{\max _{i}\left(\chi_{i}\right) \rho\left(X_{2}^{-1} M_{2}^{-1}\right)} .
$$

According to Theorem 1,

$$
\sqrt{\min _{i}\left(\chi_{i}\right) \rho\left(X_{2}^{-1} M_{2}^{-1}\right)} \leqslant R_{0} \leqslant \sqrt{\max _{i}\left(\chi_{i}\right) \rho\left(X_{2}^{-1} M_{2}^{-1}\right)}+\max _{i}\left(q_{1 i}\right) .
$$

This finishes the proof. 
Proof of Corollary 1. By the assumptions in (33), we have $\min _{i}\left(\chi_{i}\right)=\chi=\max _{i}\left(\chi_{i}\right)$. Corollary follows from Theorem 2 .

Proof of Theorem 3. According to Theorem 6,

$$
\frac{1}{\max _{i}\left(d_{2 i}+\varepsilon_{2 i}\right) \max _{i}\left(d_{2 i}+\gamma_{2 i}+\mu_{2 i}\right)} \leqslant \rho\left(X_{2}^{-1} M_{2}^{-1}\right) \leqslant \frac{1}{\min _{i}\left(d_{2 i}+\varepsilon_{2 i}\right) \min _{i}\left(d_{2 i}+\gamma_{2 i}+\mu_{2 i}\right)} .
$$

By Theorem 2, we have

$$
\sqrt{\frac{\min _{i}\left(\chi_{i}\right)}{\max _{i}\left(d_{2 i}+\varepsilon_{2 i}\right) \max _{i}\left(d_{2 i}+\gamma_{2 i}+\mu_{2 i}\right)}} \leqslant R_{0} \leqslant \sqrt{\frac{\max _{i}\left(\chi_{i}\right)}{\left.\min _{i}\left(d_{2 i}+\varepsilon_{2 i}\right) \min _{(} d_{2 i}+\gamma_{2 i}+\mu_{2 i}\right)}}+\max _{i}\left(q_{1 i}\right) .
$$

This finishes the proof.

Proof of Corollary 2. By the conditions in (33) and (37), we have

$\frac{\min _{i}\left(\chi_{i}\right)}{\max _{i}\left(d_{2 i}+\varepsilon_{2 i}\right) \max _{i}\left(d_{2 i}+\gamma_{2 i}+\mu_{2 i}\right)}=\frac{\chi}{\left(d_{2}+\varepsilon_{2}\right)\left(d_{2}+\gamma_{2}+\mu_{2}\right)}=\frac{\max _{i}\left(\chi_{i}\right)}{\min _{i}\left(d_{2 i}+\varepsilon_{2 i}\right) \min _{(}\left(d_{2 i}+\gamma_{2 i}+\mu_{2 i}\right)}$.

Corollary follows from Theorem 3.

Theorem 4. If both $A$ and $B$ are non-negative square matrices, then $\rho(A) \leq \rho(A+B)$.

Proof. Recall that the Gelfand's formula is that

$$
\rho(A)=\lim _{k \rightarrow \infty}\left\|A^{k}\right\|^{\frac{1}{k}}
$$

for any matrix norm $\|\cdot\|$. If $A, B$ are both non-negative, then $A \leq A+B$. Hence, $0 \leq A^{k} \leq(A+B)^{k}$ for any $k \in \mathbb{N}$. By the property of matrix norm, $0 \leq\left\|A^{k}\right\| \leq\left\|(A+B)^{k}\right\|$. Thus,

$$
0 \leq \lim _{k \rightarrow \infty}\left\|A^{k}\right\|^{\frac{1}{k}} \leq \lim _{k \rightarrow \infty}\left\|(A+B)^{k}\right\|^{\frac{1}{k}} .
$$

The theorem follows from the Gelfand's formula.

Theorem 5. For the model presented in Section 2.2 (Equations (18) through (23)), a unique nonnegative solution for total number of species $k$ individuals in node $i$ at DFE exists.

Proof. To solve the total number of species $k$ individuals in each node at DFE, we need to solve the following system of equations.

$$
\mathcal{W}\left[\begin{array}{llll}
N_{k 1}^{*} & N_{k 2}^{*} & \cdots & N_{k n}^{*}
\end{array}\right]^{T}=\left[\begin{array}{llll}
r_{k 1} & r_{k 2} & \cdots & r_{k n}
\end{array}\right]^{T},
$$

where

$$
\mathcal{W}=\left[\begin{array}{cccc}
d_{k 1}+\sum_{j=2}^{n} \omega_{k 1 j} & -\omega_{k 21} & \cdots & -\omega_{k n 1} \\
-\omega_{k 12} & d_{k 2}+\sum_{j=1, j \neq 2}^{n} \omega_{k 2 j} & \cdots & -\omega_{k n 2} \\
\cdots & \cdots & \cdots & \cdots \\
-\omega_{k 1 n} & -\omega_{k 2 n} & \cdots & d_{k n}+\sum_{j=1}^{n-1} \omega_{k n j}
\end{array}\right]
$$

The variable vector $\left[\begin{array}{ccc}N_{k 1}^{*} & N_{k 2}^{*} \cdots N_{k n}^{*}\end{array}\right]^{T}$ is to be solved. We note that $\mathcal{W}$ is a diagonal dominant matrix of its column entries [41], i.e., $\mathcal{W}_{i i} \geqslant \sum_{i=1, i \neq j}^{n} \mathcal{W}_{i j}$, for all $i$, where $\mathcal{W}_{i j}$ is the $(i, j)$ entry of $\mathcal{W}$. By Theorem 1 in page 654 of [41], $\mathcal{W}$ is invertible. Moreover, by Theorem 7 in appendix, $\mathcal{W}^{-1}$ is nonnegative. Thus, there exists a unique nonnegative solution for the system of equations (45). The unique nonnegative solution is

$$
\left[\begin{array}{llll}
N_{k 1}^{*} & N_{k 2}^{*} & \cdots & N_{k n}^{*}
\end{array}\right]^{T}=\left[\begin{array}{llll}
N_{k 1}^{0} & N_{k 2}^{0} & \cdots & N_{k n}^{0}
\end{array}\right]^{T}=\mathcal{W}^{-1}\left[\begin{array}{llll}
r_{k 1} & r_{k 2} & \cdots & r_{k n}
\end{array}\right]^{T} .
$$


Theorem 6. Let $A_{k}(k=1,2, \cdots, m)$ be an $n \times n$ diagonal dominant matrix with $A_{k}^{-1} \geq 0$. Denote the $(i, j)$ entry of $A_{k}$ by $a_{k i j}$. Let $a_{k}^{L}=\min _{j}\left(\sum_{i} a_{k i j}\right)>0, a_{k}^{H}=\max _{j}\left(\sum_{i} a_{k i j}\right)$, where $j=1,2, \cdots, n$, then

$$
\prod_{k=1}^{m} \frac{1}{a_{k}^{H}} \leqslant \rho\left(\prod_{k=1}^{m} A_{k}^{-1}\right) \leqslant \prod_{k=1}^{m} \frac{1}{a_{k}^{L}} .
$$

Proof. Clearly, $0 \leq a_{k}^{L} \mathcal{C} \leqslant \mathcal{C} A_{k} \leqslant a_{k}^{H} \mathcal{C}$, where $\mathcal{C}=\left[\begin{array}{llll}1 & 1 & \cdots & 1 \times n\end{array}\right]_{1 \times n}$. Since $A_{k}^{-1} \geqslant 0$, we obtain

$$
0 \leq \frac{\mathcal{C}}{a_{k}^{H}} \leq \mathcal{C} A_{k}^{-1} \leq \frac{\mathcal{C}}{a_{k}^{L}}
$$

Similarly,

$$
0 \leq \frac{\mathcal{C}}{a_{k}^{H} a_{k-1}^{H}} \leq \frac{\mathcal{C} A_{k-1}^{-1}}{a_{k}^{H}} \leq \mathcal{C} A_{k}^{-1} A_{k-1}^{-1} \leq \frac{\mathcal{C} A_{k-1}^{-1}}{a_{k}^{L}} \leq \frac{\mathcal{C}}{a_{k}^{L} a_{k-1}^{L}} .
$$

Following the same argument,

$$
\prod_{k=1}^{m} \frac{1}{a_{k}^{H}} \mathcal{C} \leqslant \mathcal{C} \prod_{k=1}^{m} A_{k}^{-1} \leqslant \prod_{k=1}^{m} \frac{1}{a_{k}^{L}} \mathcal{C} .
$$

By Corollary 1 in [40], any $n \times n$ nonnegative matrix $A$ satisfies:

$$
\min _{j}\left(\sum_{i=1}^{n} a_{i j}\right) \leqslant \rho(A) \leqslant \max _{j}\left(\sum_{i=1}^{n} a_{i j}\right) .
$$

Because the entries of $\mathcal{C} \prod_{k=1}^{m} A_{k}^{-1}$ is the sum of each column of matrix $\prod_{k=1}^{m} A_{k}^{-1}$, by Inequality (46),

$$
\prod_{k=1}^{m} \frac{1}{a_{k}^{H}} \leqslant \rho\left(\prod_{k=1}^{m} A_{k}^{-1}\right) \leqslant \prod_{k=1}^{m} \frac{1}{a_{k}^{L}} .
$$

Theorem 7. Matrices $M_{k}$ and $X_{k}$ in (24) are invertible, and $M_{k}$ and $X_{k}$ are nonnegative. Moreover, Matrices $M_{k}^{-1}$ and $X_{k}^{-1}$ are nonnegative matrices.

Proof. Note that $M_{k}$ is a diagonal dominant matrix of its column entries. By Theorem 1 in page 654 of [41], $M_{k}$ and $X_{k}$ are invertible. We now prove that $M_{k}^{-1}$ is nonnegative. Matrix $M_{k}$ can be rewritten as follows.

$$
M_{k}=\left[\begin{array}{cccc}
\zeta_{k 1} & -\omega_{k 21} & \cdots & -\omega_{k n 1} \\
-\omega_{k 12} & \zeta_{k 2} & \cdots & -\omega_{k n 2} \\
\cdots & \cdots & \cdots & \cdots \\
-\omega_{k 1 n} & -\omega_{k 2 n} & \cdots & \zeta_{k n}
\end{array}\right]=\oplus_{i=1}^{n} \zeta_{k i}-\left[\begin{array}{cccc}
0 & \omega_{k 21} & \cdots & \omega_{k n 1} \\
\omega_{k 12} & 0 & \cdots & \omega_{k n 2} \\
\cdots & \cdots & \cdots & \cdots \\
\omega_{k 1 n} & \omega_{k 2 n} & \cdots & 0
\end{array}\right]=: G-H .
$$

Consequently,

$$
G^{-1}=\oplus_{i=1}^{n} \zeta_{k i}^{-1} \quad \text { and } \quad G^{-1} H=\left[\begin{array}{cccc}
0 & \omega_{k 21} \zeta_{k 1}^{-1} & \cdots & \omega_{k n 1} \zeta_{k 1}^{-1} \\
\omega_{k 12} \zeta_{k 2}^{-1} & 0 & \cdots & \omega_{k n 2} \zeta_{k 2}^{-1} \\
\cdots & \cdots & \cdots & \cdots \\
\omega_{k 1 n} \zeta_{k n}^{-1} & \omega_{k 2 n} \zeta_{k n}^{-1} & \cdots & 0
\end{array}\right]
$$

Moreover, $0<\sum_{j=1}^{n}\left(G^{-1} H\right)_{i j}<1$, for all $i$. Hence, $\rho\left(G^{-1} H\right)<1$, i.e., $G^{-1} H$ is convergent (see [28]). Obviously, $G^{-1} \geqslant 0$, and $G^{-1} H \geqslant 0$. By Theorem 1 in [28], $M_{k}$ is an M-matrix and $M_{k}^{-1} \geqslant 0$. By the same argument, $X_{k}$ is an M-matrix and $X_{k}^{-1} \geqslant 0$. This finishes the proof. 
Network-based RVF meta- population model

Aedes population model

$$
\begin{aligned}
\frac{\mathrm{d} P_{1 i}}{\mathrm{~d} t} & =b_{1 i}\left(N_{1 i}-q_{1 i} I_{1 i}\right)-\theta_{1 i} P_{1 i} \\
\frac{\mathrm{d} Q_{1 i}}{\mathrm{~d} t} & =b_{1 i} q_{1 i} I_{1 i}-\theta_{1 i} Q_{1 i} \\
\frac{\mathrm{d} S_{1 i}}{\mathrm{~d} t} & =\theta_{1 i} P_{1 i}-d_{1 i} S_{1 i} N_{1 i} / K_{1}-\beta_{21 i} S_{1 i} I_{2 i} / N_{2 i} \\
\frac{\mathrm{d} E_{1 i}}{\mathrm{~d} t} & =\beta_{21 i} S_{1 i} I_{2 i} / N_{2 i}-\varepsilon_{1 i} E_{1 i}-d_{1 i} E_{1 i} N_{1 i} / K_{1} \\
\frac{\mathrm{d} I_{1 i}}{\mathrm{~d} t} & =\theta_{1 i} Q_{1 i}+\varepsilon_{1 i} E_{1 i}-d_{1 i} I_{1 i} N_{1 i} / K_{1} \\
\frac{\mathrm{d} N_{1 i}}{\mathrm{~d} t} & =\theta_{1 i}\left(P_{1 i}+Q_{1 i}\right)-d_{1 i} N_{1 i} N_{1 i} / K_{1}
\end{aligned}
$$

Culex population model

$$
\begin{aligned}
\frac{\mathrm{d} P_{3 i}}{\mathrm{~d} t} & =b_{3 i} N_{3 i}-\theta_{3 i} P_{3 i} \\
\frac{\mathrm{d} S_{3 i}}{\mathrm{~d} t} & =\theta_{3 i} P_{3 i}-\beta_{23 i} S_{3 i} I_{2 i} / N_{2 i}-d_{3 i} S_{3 i} N_{3 i} / K_{3} \\
\frac{\mathrm{d} E_{3 i}}{\mathrm{~d} t} & =\beta_{23 i} S_{3 i} I_{2 i} / N_{2 i}-\varepsilon_{3 i} E_{3 i}-d_{3 i} E_{3 i} N_{3 i} / K_{3} \\
\frac{\mathrm{d} I_{3 i}}{\mathrm{~d} t} & =\varepsilon_{3 i} E_{3 i}-d_{3 i} I_{3 i} N_{3 i} / K_{3} \\
\frac{\mathrm{d} N_{3 i}}{\mathrm{~d} t} & =\theta_{3 i} P_{3 i}-d_{3 i} N_{3 i} N_{3 i} / K_{3}
\end{aligned}
$$

Livestock population model

$$
\begin{aligned}
\frac{\mathrm{d} S_{2 i}}{\mathrm{~d} t} & =r_{2 i}-\beta_{12 i} S_{2 i} I_{1 i} / N_{1 i}-\beta_{32 i} S_{2 i} I_{3 i} / N_{3 i}-d_{2 i} S_{2 i}+\sum_{j=1, j \neq i}^{n} \omega_{2 j i} S_{2 j}-\sum_{j=1, j \neq i}^{n} \omega_{2 i j} S_{2 i} \\
\frac{\mathrm{d} E_{2 i}}{\mathrm{~d} t} & =\beta_{12 i} S_{2 i} I_{1 i} / N_{1 i}+\beta_{32 i} S_{2 i} I_{3 i} / N_{3 i}-\varepsilon_{2 i} E_{2 i}-d_{2 i} E_{2 i}+\sum_{j=1, j \neq i}^{n} \omega_{2 j i} E_{2 j}-\sum_{j=1, j \neq i}^{n} \omega_{2 i j} E_{2 i} \\
\frac{\mathrm{d} I_{2 i}}{\mathrm{~d} t} & =\varepsilon_{2 i} E_{2 i}-\gamma_{2 i} I_{2 i}-\mu_{2 i} I_{2 i}-d_{2 i} I_{2 i}+\sum_{j=1, j \neq i}^{n} \omega_{2 j i} I_{2 j}-\sum_{j=1, j \neq i}^{n} \omega_{2 i j} I_{2 i} \\
\frac{\mathrm{d} R_{2 i}}{\mathrm{~d} t} & =\gamma_{2 i} I_{2 i}-d_{2 i} R_{2 i}+\sum_{j=1, j \neq i}^{n} \omega_{2 j i} R_{2 j}-\sum_{j=1, j \neq i}^{n} \omega_{2 i j} R_{2 i} \\
\frac{\mathrm{d} N_{2 i}}{\mathrm{~d} t} & =r_{2 i}-\mu_{2 i} I_{2 i}-d_{2 i} N_{2 i}+\sum_{j=1, j \neq i}^{n} \omega_{2 j i} N_{2 j}-\sum_{j=1, j \neq i}^{n} \omega_{2 i j} N_{2 i}
\end{aligned}
$$

Human population model

$$
\begin{aligned}
\frac{\mathrm{d} S_{4 i}}{\mathrm{~d} t} & =b_{4 i} N_{4 i}-\beta_{14 i} S_{4 i} I_{1 i} / N_{1 i}-\beta_{24 i} S_{4 i} I_{2 i} / N_{2 i}-\beta_{34 i} S_{4 i} I_{3 i} / N_{3 i}-d_{4 i} S_{4 i} N_{4 i} / K_{4} \\
\frac{\mathrm{d} E_{4 i}}{\mathrm{~d} t} & =\beta_{14 i} S_{4 i} I_{1 i} / N_{1 i}+\beta_{24 i} S_{4 i} I_{2 i} / N_{2 i}+\beta_{34 i} S_{4 i} I_{3 i} / N_{3 i}-\varepsilon_{4 i} E_{4 i}-d_{4 i} E_{4 i} N_{4 i} / K_{4} \\
\frac{\mathrm{d} I_{4 i}}{\mathrm{~d} t} & =\varepsilon_{4 i} E_{4 i}-\gamma_{4 i} I_{4 i}-\mu_{4 i} I_{4 i}-d_{4 i} I_{4 i} N_{4 i} / K_{4}
\end{aligned}
$$




$$
\begin{aligned}
& \frac{\mathrm{d} R_{4 i}}{\mathrm{~d} t}=\gamma_{4 i} I_{4 i}-d_{4 i} R_{4 i} N_{4 i} / K_{4} \\
& \frac{\mathrm{d} N_{4 i}}{\mathrm{~d} t}=b_{4 i} N_{4 i}-\mu_{4 i} I_{4 i}-d_{4 i} N_{4 i} N_{4 i} / K_{4}
\end{aligned}
$$

\begin{tabular}{|c|c|c|c|c|}
\hline Parameter & Description & $\begin{array}{ll}\text { Range } & \text { or } \\
\text { value } & \end{array}$ & Units & Source \\
\hline$\beta_{12}$ & contact rate: Aedes to livestock & $(0.0021,0.2762)$ & $1 /$ day & {$[42,43,44,45,46,47,48]$} \\
\hline$\beta_{21}$ & contact rate: livestock to Aedes & $(0.0021,0.2429)$ & $1 /$ day & {$[42,43,44,45,46,49]$} \\
\hline$\beta_{23}$ & contact rate: livestock to Culex & $(0.0000,0.3200)$ & $1 /$ day & {$[43,44,45,46,49,50]$} \\
\hline$\beta_{32}$ & contact rate: Culex to livestock & $(0.0000,0.096)$ & $1 /$ day & {$[43,44,45,46,50]$} \\
\hline$\beta_{14}$ & contact rate: Aedes to humans & & $1 /$ day & \\
\hline$\beta_{24}$ & contact rate: livestock to humans & & $1 /$ day & \\
\hline$\beta_{34}$ & contact rate: Culex to humans & & $1 /$ day & \\
\hline $1 / \gamma_{2}$ & recover rate in livestock & $(2,5)$ & days & {$[51]$} \\
\hline $1 / \gamma_{4}$ & recover period in humans & $(4,7)$ & days & {$[52]$} \\
\hline $1 / d_{1}$ & longevity of Aedes mosquitoes & $(3,60)$ & days & {$[53,54,46]$} \\
\hline $1 / d_{2}$ & longevity of livestock & $(360,3600)$ & days & {$[55]$} \\
\hline $1 / d_{3}$ & longevity of Culex mosquitoes & $(3,60)$ & days & {$[53,54,46]$} \\
\hline $1 / d_{4}$ & longevity of humans & & days & \\
\hline$b_{1}$ & egg laying rate of Aedes mosquitoes & & $1 /$ day & {$[53,54,46]$} \\
\hline$b_{3}$ & egg laying rate of Culex mosquitoes & & $1 /$ day & {$[53,54,46]$} \\
\hline$b_{4}$ & birth rate of humans & & $1 /$ day & \\
\hline $1 / \epsilon_{1}$ & $\begin{array}{l}\text { incubation period in Aedes } \\
\text { mosquitoes }\end{array}$ & $(4,8)$ & days & {$[47]$} \\
\hline $1 / \epsilon_{2}$ & incubation period in livestock & $(2,6)$ & days & {$[56]$} \\
\hline $1 / \epsilon_{3}$ & $\begin{array}{l}\text { incubation period in Culex } \\
\text { mosquitoes }\end{array}$ & $(4,8)$ & days & {$[47]$} \\
\hline $1 / \epsilon_{4}$ & incubation period in humans & $(2,6)$ & days & {$[52]$} \\
\hline$\mu_{2}$ & mortality rate in livestock & $(0.025,0.1)$ & $1 /$ day & {$[51,56]$} \\
\hline$q_{1}$ & $\begin{array}{l}\text { transovarial transmission rate in } \\
\text { Aedes mosquitoes }\end{array}$ & $(0,0.1)$ & $1 /$ day & {$[57]$} \\
\hline $1 / \theta_{1}$ & $\begin{array}{l}\text { development period of Aedes } \\
\text { mosquitoes }\end{array}$ & $(5,15)$ & days & {$[46]$} \\
\hline $1 / \theta_{3}$ & $\begin{array}{l}\text { development period of Culex } \\
\text { mosquitoes }\end{array}$ & $(5,15)$ & days & {$[46]$} \\
\hline$K_{1}$ & $\begin{array}{l}\text { carrying capacity of Aedes } \\
\text { mosquitoes }\end{array}$ & 10000 & & \\
\hline$K_{3}$ & $\begin{array}{l}\text { carrying capacity of Culex } \\
\text { mosquitoes }\end{array}$ & 10000 & & \\
\hline$K_{4}$ & carrying capacity of humans & 100000 & & \\
\hline$r_{2 i}$ & livestock recruitment rate & 1 & $1 /$ day & {$[52]$} \\
\hline$\omega_{2 i j}$ & $\begin{array}{l}\text { livestock movement rate from node } \\
i \text { to node } j\end{array}$ & $\left(0, \frac{1}{n}\right)$ & $1 /$ day & \\
\hline
\end{tabular}

Table 2: Parameters in the model omitting the node index 